\title{
Modeling mass transfer and reaction of dilute solutes in a ternary phase system by the lattice Boltzmann method
}

\author{
Yu-Hang Fu, ${ }^{1}$ Lin Bai, ${ }^{1}$ Kai-Hong Luo, ${ }^{2}$ Yong Jin, ${ }^{1}$ and Yi Cheng ${ }^{1, *}$ \\ ${ }^{1}$ Department of Chemical Engineering, Tsinghua University, Beijing 100084, P.R. China \\ ${ }^{2}$ Department of Mechanical Engineering, University College London, Torrington Place, London WC1E 7JE, United Kingdom
}

(Received 1 January 2017; published 14 April 2017)

\begin{abstract}
In this work, we propose a general approach for modeling mass transfer and reaction of dilute solute(s) in incompressible three-phase flows by introducing a collision operator in lattice Boltzmann (LB) method. An LB equation was used to simulate the solute dynamics among three different fluids, in which the newly expanded collision operator was used to depict the interface behavior of dilute solute(s). The multiscale analysis showed that the presented model can recover the macroscopic transport equations derived from the Maxwell-Stefan equation for dilute solutes in three-phase systems. Compared with the analytical equation of state of solute and dynamic behavior, these results are proven to constitute a generalized framework to simulate solute distributions in three-phase flows, including compound soluble in one phase, compound adsorbed on single-interface, compound in two phases, and solute soluble in three phases. Moreover, numerical simulations of benchmark cases, such as phase decomposition, multilayered planar interfaces, and liquid lens, were performed to test the stability and efficiency of the model. Finally, the multiphase mass transfer and reaction in Janus droplet transport in a straight microchannel were well reproduced.
\end{abstract}

DOI: 10.1103/PhysRevE.95.043304

\section{INTRODUCTION}

Microfluidic technology has drawn much attention in many scientific areas and engineering applications, in which three-phase flows usually involve complex interactions among phases. For example, in droplet-based microfluidics, emulsions consisting of multiple species can be precisely controlled to generate, coalesce, and breakup [1,2]. One of the typical examples is the manufacture of the multi- and double-emulsion in microfluidic devices with desired droplet size and exquisite structure, leading to a rich variety of applications such as drug release, delivery, and diagnosis [3] as well as the synthesis of functional particles [4,5]. In some of the above-mentioned processes, species transport across the phase interface is often encountered, which undoubtedly plays a key role in the overall performance of the underlying process. This leads to widespread research interest in academia to investigate the interface transport phenomena at microscale using analytical methods or experimental techniques for rational design of microfluidic devices.

Due to the limited capability of most experimental techniques to perform the in situ measurement in microfluidics, analytical methods are advantageous in gaining in-depth knowledge on the complicated multiphase transport phenomena at different scales, though it is still challenging to appropriately model the interface rupturing and merging or resolve the description of the complex interactions among multiphase fluids in microchannels. As for the current interest on modeling the interface dynamics of a multiphase system, great efforts have been made to develop computational technologies, such as the level set method [6-8], volume of fluid (VOF) method [9], diffuse interface model [10,11],

\footnotetext{
*Corresponding author: Department of Chemical Engineering, Tsinghua University, Beijing 100084, P.R. China; yicheng@tsinghua.edu.cn.
}

and phase-field model [12]. However, few numerical studies have concentrated on mass transfer or mixing performance of inert or reactive species in multiphase flows, especially for more than two-phase flows. For example, considering the droplet-based microreactor, the extraction process or flow chemistry based on microfluidics usually contains the nonideal solutes that are insoluble or partially soluble in the dispersed microdroplet or not. The reaction reagent would transfer across the interface of phases. Therefore, it is fundamental to understand the hydrodynamics and distribution of the nonideal systems containing solutes when mass transfer takes place at the interface in complex multiphase flows. Several numerical models have been proposed to describe the inert solute or reactive species mass transfer in multiphase flows without phase change. Normally, the solute behavior is governed by the diffusion-convection equation in a single-phase domain coupled with a species distribution law (i.e., Henry's law) at the interface, assuming an equilibrium state at the interface [13-16]. The earliest attempt for such mass transfer modeling is the case for a rising droplet by the VOF method. This model is largely based on the continuous change of the concentration of the solutes at interface $[17,18]$. The numerical approach treats the discontinuity of the solute concentration at interface by a single-field approach, where this discontinuous physical property at interface still challenges the stability and capability of model predictions. The approaches of moving mesh technology [19], level set method [20], transformation technique [21] were used to improve the computational performance (i.e., stability and accuracy). The species concentration could be calculated separately in each phase domain, resulting in an affordable computational load in complex flows.

Due to the strength including algorithmic simplicity, geometric flexibility and parallel efficiency, lattice Boltzmann method (LBM) has found numerous applications in multiphase flow modeling $[22,23]$. The basic equation in LBM describes the distribution functions of an assembly of particles to mimic the microscopic interactions between fluids particles governed 
by the Boltzmann equation [24]. The actions of streaming and collision of these particles account for the flow hydrodynamics, ensuring the recovery of macroscopic conservation laws. For the modeling of multiphase flows, several LB formulations have been established. They are commonly categorized into the color gradient model [25], the pseudopotential model [26], the free-energy model [27], and the phase-field model [28]. Among them, the color-gradient model was first introduced to three-phase flows by Dupin et al. [29] and their next work [30], in which the multicomponent flows with low density ratio can be simulated. Leclaire et al. [31] developed an enhanced color-gradient model with improved numerical stability, where the collision function incorporates three subparts (i.e., singlephase operator, perturbation operator, recoloring operator). It was able to simulate immiscible multiphase flows with high density ratio up to $\mathrm{O}(1000)$ and viscosity ratio up to $\mathrm{O}(100)$, and a generalized color gradient force was introduced for more than two-phase systems. Pseudopotential models [26,32] were also able to simulate multiphase flows, although their capability for modeling multicomponent multiphase flows have not been fully explored. Shi et al. [33] applied a lattice Boltzmann flux solver for the three-component Cahn-Hilliard model, in which the interactions among three fluids were carefully examined. Chen et al. [34] applied the three order parameters to model fluid interactions by self-consistent forces. Semprebon et al. [35] extended the free-energy model to ternary phases, where the fluid-fluid surface tension and the solid surface contact angles can be specified. Liang et al. [36] expanded the phase-field model into three-phase fluid systems, but the model is restricted to a limited set of interface tension of each phase. One can find more details of ternary LB models in a recent review article [37].

Several attempts were carried out to capture the mass transfer or mixing problems of multicomponent multiphase mixtures using LBM. The molecular interaction was first introduced based on the pseudopotential model [38], and the thermodynamic inconsistency was found in the following research [39]. A new collision operator was established for multicomponent gaseous mixtures by Luo and Girimaji [40], and their model eventually allowed detonation modeling accounting for different molecular weights [41], large density ratios [42], and temperature gradients [43]. The thermodynamic consistency could be guaranteed with an additional parameter. Although some nonlocal terms determined by multiscale analysis were to be numerically corrected, a wide span of complex phenomena associated with multicomponent mixture could be successfully predicted. Since these models combined the thermodynamics and transport equations, the complexity of the algorithm would grow rapidly with the number of compounds. In chemistry and chemical engineering, the assumption of neglecting the interactions between different solutes and solvents has been commonly employed, resulting in describing the solvent and solute separately with different governing equations [44]. Modeling the hydrodynamic behavior of dilute solutes in interphase mass transfer situations is of considerable importance not only in chemical processes but also for physical understanding of the interface phenomenon.

Regarding solute modeling, the earliest attempts for modeling inert or reactive solutes in single-phase flow based on the diffusion convection equation could be attributed to
Ref. [45]. The schemes of single relaxation time (SRT), two relaxation time (TRT), and multiple relaxation time (MRT) models were developed with second-order accurate transport equations for the solute species and the anisotropic diffusion was characterized [45]. The reactive source term was added in the governing equations of reactive species for fast kinetic reactions [46-48]. In our previous work, a framework was established for modeling multiphase solute mass transfer and thermodynamic properties based on the Maxwell-Stefan equation [49]. A Taylor-expansion analysis of the collision operator describing the interface profile in a binary system was well discussed. Based on our previous work, we present the newly expanded collision operator for depicting the generalized framework of dilute solutes transport in three-phase systems. The behavior of four kinds of dilute species, i.e., single-phase soluble compound, single-interface adsorbed compound, two-phase soluble compound, and threephase soluble compound are well discussed.

To sum up, this paper is organized as follows. In Sec. II, we present the implementation of LB model and the derivation of the LB model of the dilute solutes is discussed, where the results of Chapman-Enskog analysis are compared to Maxwell-Stefan equations. In Sec. III, a series of numerical cases are presented to test the performance of this newly established model. Finally, conclusions are drawn at the end.

\section{LATTICE BOLTZMANN METHOD IMPLEMENTATION}

This section describes the details of the numerical implementation of the mass transfer of dilute species in multiphase flows by further developing the lattice Boltzmann method. Due to the nature of the highly dilute species, the motion of the bulk phase can be decoupled from the dilute species. In other words, the equations can be solved and processed separately without the contribution of the dilute species. As pointed out in the Introduction, much attention has been paid for ternary flow systems, in which the color-gradient model [29-31,50,51], pseudopotential model [26,32,52], and free-energy model $[33,36]$ can be implemented for the bulk phase. In the following part, the mass transfer of dilute species is processed and the concentration field is revealed based on the microscopic values such as the distribution of each phase and the velocity of the system calculated from the bulk phase at each time step. The first part of this section illustrates a ternary color-gradient model of an LBM scheme for bulk multiphase flow. The second part describes the LBM algorithm for dilute solute in a multiphase system and highlights the meaning of the added collision operator for interface profile with the Maxwell-Stefan equation. The organization of the two parts is shown in Fig. 1.

\section{A. Ternary Color-gradient model for bulk phase flow}

Color-gradient-based lattice Boltzmann models use Red, Blue, and Green particle distribution functions (PDFs) $f_{i}^{R}, f_{i}^{B}$, and $f_{i}^{G}$ to represent the three fluids. The total PDF is defined as $f_{i}=f_{i}^{R}+f_{i}^{B}+f_{i}^{G}$. Each of the color phases obeys the governing equations proposed as

$$
f_{i}^{k}\left(x_{\alpha}+e_{i \alpha} \delta t, t+\delta t\right)=f_{i}^{k}\left(x_{\alpha}, t\right)+\Omega_{i}^{k},
$$


where the superscript $k=R, B$,or $G$ represent the color phases (Red, Blue, or Green), $f_{i}^{k}\left(x_{\alpha}, t\right)$ is the particle distribution function and $\delta t$ is the time step. The lattice Boltzmann models discretize the Boltzmann dynamics in space $\left(x_{\alpha}\right)$ and time $(t)$ with the help of the lattice velocities $\left(e_{i \alpha}\right)$. Based on the discretization, the lattice schemes are classified in a $2 \mathrm{D}$ or 3D model with different total number of particle velocities, whereas each LBM scheme needs to obey the conservation of the mechanical flux tensors. Therefore, the macroscopic quantities (density and velocity) can be calculated from the particle distribution function as

$$
\begin{gathered}
\rho_{k}=\sum_{i} f_{i}^{k}, \\
\rho u_{\alpha}=\sum_{i} \sum_{k} f_{i}^{k} e_{i \alpha},
\end{gathered}
$$

where $i$ th is the velocity direction, $\rho_{k}$ is the density of phase $k$, total density is $\rho=\sum_{k} \rho_{k}$, and $u_{\alpha}$ is the local velocity vector. For the two-dimensional nine-velocity (D2Q9) model, the basic coefficients are given in Table I.

The PDF of each phase takes the collision and streaming operators for fluid mechanics. The collision operator of colorgradient model contains the separation for each fluid and momentum exchange in single-phase and multiphase fields. Specifically, the collision operator results in the combination
TABLE I. Basic parameters for color-gradient model in D2Q9.

\begin{tabular}{lccc}
\hline \hline & Center & Lateral face & Diagonal \\
\hline Coordinate & $(0,0)$ & $(0, \pm 1) \operatorname{or}( \pm 1,0)$ & $( \pm 1, \pm 1)$ \\
Velocity directions & $i=0$ & $i=1,2,3,4$ & $i=5,6,7,8$ \\
$w_{i}$ & $4 / 9$ & $1 / 9$ & $1 / 36$ \\
$\phi_{k}^{i}$ & $\alpha_{k}$ & $\left(1-\alpha_{k}\right) / 5$ & $\left(1-\alpha_{k}\right) / 20$ \\
$B_{i}$ & $-4 / 27$ & $2 / 27$ & $5 / 108$ \\
$c_{s}$ & & $\sqrt{3} / 3$ with $\delta x=\delta t=1$ \\
\hline \hline
\end{tabular}

of three suboperators [31,51]:

$$
\Omega_{i}^{k}=\left(\Omega_{i}^{k}\right)^{3}\left[\left(\Omega_{i}^{k}\right)^{1}+\left(\Omega_{i}^{k}\right)^{2}\right],
$$

where $\left(\Omega_{i}^{k}\right)^{1}$ is the single-phase collision operator, and $\left(\Omega_{i}^{k}\right)^{2}$ and $\left(\Omega_{i}^{k}\right)^{3}$ are multiphase collision operators. The former one, $\left(\Omega_{i}^{k}\right)^{2}$, is called perturbation operator, generating an interfacial tensor at the mixed interfacial region, and the latter one, $\left(\Omega_{i}^{k}\right)^{3}$, is called recoloring operator, controlling alterable interface thickness and conserves the phase segregation.

The single-phase collision operator employs the single relaxation time to simplify the collision operator with the help of the local equilibrium called Bhatnagar-Gross-Krook (BGK) approximation [53]:

$$
\left(\Omega_{i}^{k}\right)^{1}=-\frac{1}{\bar{\tau}}\left(f_{i}^{k}-f_{i}^{k(\mathrm{eq})}\right)
$$

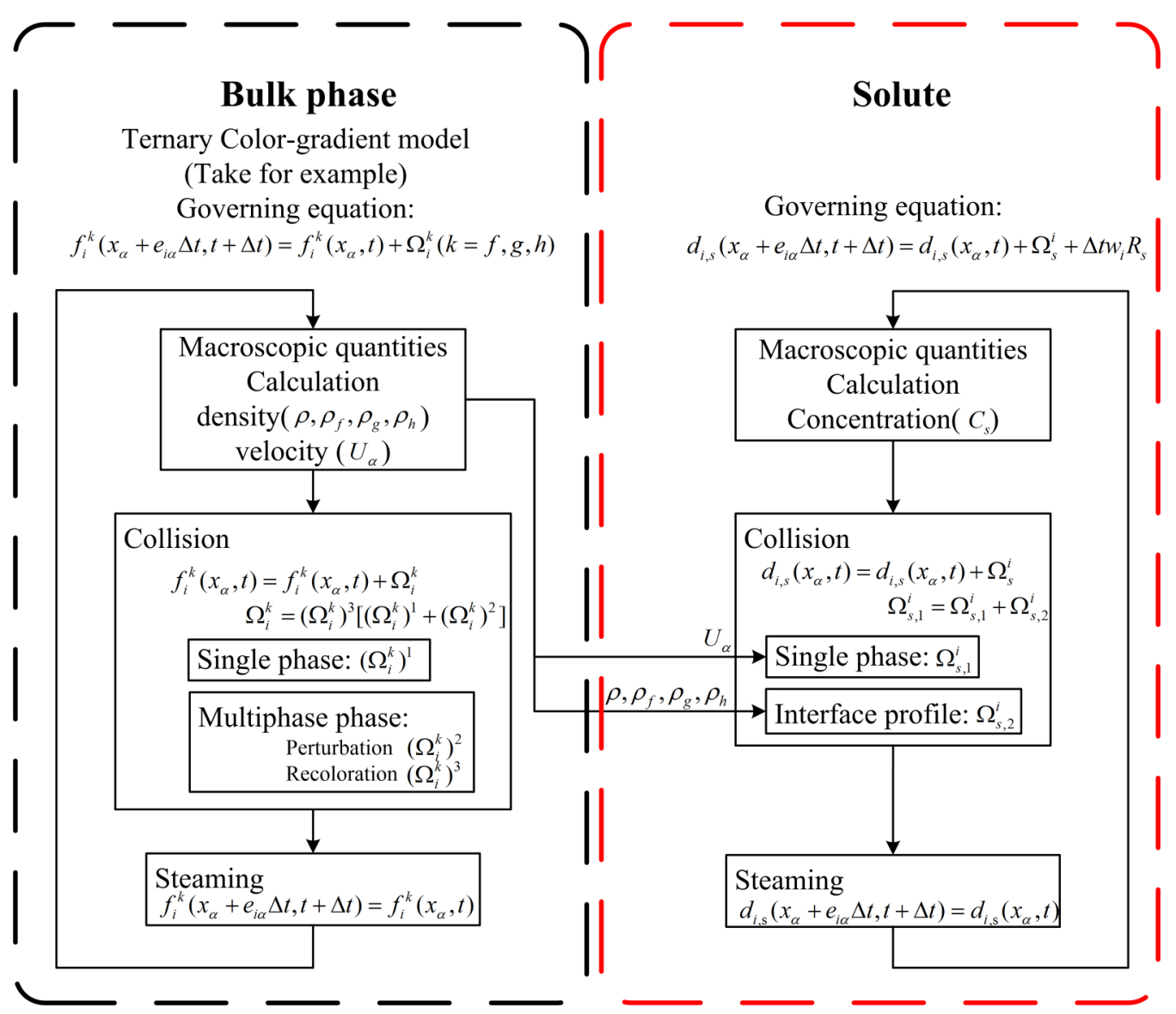

FIG. 1. Program chart of LBM scheme to model the mass transfer of the dilute species in multiphase flows. Bulk phase equations are described by a color-gradient LBM model in Sec. II A and the solute equations are given in Sec. II B. 
where $\bar{\tau}$ is the average relaxation time defined as $\bar{\tau}=$ $3 \rho / \sum_{k} \frac{\rho_{k}}{v_{k}}+1 / 2$ with $v_{k}$ being the kinematic viscosity of phase $k \cdot f_{i}^{k(\mathrm{eq})}$ is the equilibrium distribution function defined by:

$$
f_{i}^{k(\mathrm{eq})}=\rho_{k}\left(\phi_{i}^{k}+w_{i}\left[\frac{3}{c^{2}} e_{i \alpha} u_{\alpha}+\frac{9 e_{i \alpha} u_{\alpha} e_{i \beta} u_{\beta}}{2 c^{4}}-\frac{3 u_{\alpha} u_{\alpha}}{2 c^{2}}\right]\right),
$$

where $c=11 . u$./t.s. composes the standard velocity in lattice units per step. Other parameters are given in Table I. $\alpha_{k}$ is a free parameter and the density ratio is taken as

$$
\lambda_{k l}=\frac{1-\alpha_{l}}{1-\alpha_{k}}
$$

Note that the relation $0<\alpha_{k}<1$ needs to be held for each fluid $k$ to avoid the negative value in the calculation, and $\alpha_{k}$ controls the sound speed of each phase $k$, thus determining the hydrodynamic pressure as

$$
p^{k}=\rho_{k} \frac{3\left(1-\alpha_{k}\right)}{5}=\rho_{k}\left(c_{s}^{k}\right)^{2}
$$

Usually, the source term is added in the particle distribution function in order to correct error items caused by the density ratio and reduce the nondesired part in hydrodynamics equations. In this paper, we set $\alpha_{k}=4 / 9$ for each phase $k$, thus providing an identical density for each phase.

The perturbation operator $\left(\Omega_{i}^{k}\right)^{2}$ takes advantage of the idea of continuum interfacial force $[51,54]$ to rebuild interfacial profile with low spurious velocity and isotropy of interface. It is chosen to take the form

$$
\left(\Omega_{i}^{k}\right)^{2}=\sum_{l, l \neq k} \frac{A_{k l}}{2}\left|F_{k l \alpha}\right|\left[w_{i} \frac{\left(F_{k l \alpha} e_{i \alpha}\right)^{2}}{\left|F_{k l \alpha}\right|^{2}}-B_{i}\right],
$$

where $B_{i}$ recovers the macroscopic limit within interface tensor given in Table I and $F_{k l \alpha}$ is the color-gradient force from the phase fractions. Although the color-gradient force could be implemented from another algorithm in Ref. [55], which results in a continuum surface force model and takes the force as an external force in interface profile. Equation (9) is constructed from a continuum surface force according to a diffuse-interface theory without any additional assumption [51,54]. In order to expand Eq. (9) to a more than two-phase system, a more generalized color-gradient force $F_{k l \alpha}$ is given as [31]

$$
F_{k l \alpha}=\frac{\rho_{l}}{\rho} \frac{\partial}{\partial x_{\alpha}}\left(\frac{\rho_{k}}{\rho}\right)-\frac{\rho_{k}}{\rho} \frac{\partial}{\partial x_{\alpha}}\left(\frac{\rho_{l}}{\rho}\right),
$$

where

$$
\frac{\partial}{\partial x_{\alpha}}\left(\frac{\rho_{k}}{\rho}\right)=\frac{3}{c^{2}} \sum_{i} w_{i}\left(\frac{\rho_{k}}{\rho}\right)\left(x_{\alpha}+e_{i \alpha}\right) e_{i \alpha} .
$$

This definition produces a more efficient computation of $\mathrm{O}(N)$ complexity with numerical stability, where a fourthorder isotropic discrete gradient is employed to enhance the accuracy of the ternary model as in Eq. (11). Since the color-gradient force is responsible for deriving the capillary stress tensor, one can obtain the relationship of the universal parameter $A_{k l}$ and the interfacial tension $\gamma_{k l}$ without any approximations:

$$
\gamma_{k l}=\frac{2}{9}\left(A_{k l}+A_{l k}\right) \bar{\tau} c^{4} \delta_{t}
$$

where $A_{k l}=A_{l k}$ guarantees the isotropic profile of the interface.

External force $F_{k \alpha}^{\text {ext }}$ such as the gravity or electronic fields can be incorporated into this model by adding a momentum source operator $\left(\Omega_{i}^{k}\right)^{\text {ext }}$. The implementation of the original lattice gas automata (LGA) model can be found in Ref. [56] and further improved schemes [57-59] rectify discretization effects with numerical accuracy. A comparison can be referred to Ref. [60] by Li et al. Here we note a more restricted expression by Guo et al. [58] in Eq. (13). It is worth noting that the expression of velocity should count the effect of the external force as $\rho u_{\alpha}=\sum_{i} \sum_{k}\left(f_{i}^{k} e_{i \alpha}+\frac{1}{2} F_{k \alpha}^{\text {ext }}\right)$ according to the Chapman-Enskog analysis of force term in order to recover the Navier-Stokes equations:

$$
\left(\Omega_{i}^{k}\right)^{\mathrm{ext}}=\left(1-\frac{1}{2 \tau_{k}}\right) w_{i}\left(3\left(e_{i \alpha}-u_{\alpha}\right)+9 u_{\alpha} e_{i \alpha}\right) F_{k \alpha}^{\mathrm{ext}} .
$$

Although the last sub-operator $\left(\Omega_{i}^{k}\right)^{3}$ in Eq. (14) called recoloring operator has limited physical meaning, it solves the lattice pinning problem by increasing probability of moving back to its original regions. Also, the spurious currents can be reduced and maintain a controllable sharp interface with computational efficiency. For the ternary system, the operator for each color phase is defined as

$$
\left(\Omega_{i}^{k}\right)^{3}=\frac{\rho_{k}}{\rho} \sum_{k} f_{i}^{k}+\beta_{k l}\left(\frac{\rho_{k}}{\rho}\right)\left(\frac{\rho_{l}}{\rho}\right) \cos \left(\varphi_{i}^{k l}\right) f_{i}^{\left(\rho, u_{\alpha}=0\right)}
$$

where

$$
\cos \left(\varphi_{i}^{k l}\right)=\frac{F_{k l \alpha} e_{i \alpha}}{\left|F_{k l \alpha}\right|\left|e_{i \alpha}\right|} .
$$

In the above equation, $f_{i}^{\left(\rho, u_{\alpha}=0\right)}$ denotes the equilibrium distribution function of the total mass and the velocity $u_{\alpha}=0$, and $\varphi_{i}^{k l}$ is the angle between the color-gradient force $F_{k l \alpha}$ and velocity speeds $e_{i \alpha} . \beta_{k l}$ is the segregation parameter corresponding to the interface thickness. It is noted that the equilibrium state results in a particular relation $\beta_{k l}$ due to the Neumann triangle caused by the interface tension. One can refer to Refs. [30,31] for the identical thickness of each $k-l$ interface with an improved stability.

This redistribution operator of color-gradient model gives the interface thickness as $\xi_{k l}=1 /\left(6 \beta_{k l} k\right)$, where the geometric constant $k$ is 0.1502 for the D2Q9 model following the equation

$$
2 k I_{\alpha \beta}=\sum_{i} w_{i} \frac{e_{i \alpha} e_{i \beta}}{\left|e_{i \alpha}\right|} .
$$

Combining Eqs. (1) to (15) together, the multiphase LBM for a ternary is established. After each step, the macroscopic quantities calculated from Eqs. (2) and (3) are exploited for the computation of dilute species mass transfer in Eqs. (25)-(30).

\section{B. Modeling mass transfer of dilute species in a ternary system}

The Maxwell-Stefan equation, a statistical model, turns out to be the adequate proximate framework for the mass 
transfer problem in multicomponent flows. This model is widely applied in multiphase mass transfer cases [61-63]. Considering the solute species $C_{i}$ and the total concentration $C_{t}$, this framework is based on thermodynamics that the mass flux $N_{\alpha}$ caused by the concentration gradient goes toward a minimization of the system's chemical potential. By introducing the molar fraction $x_{i}=C_{i} / C_{t}$ of species $i$, the Maxwell-Stefan equation can read as

$$
-C_{t} \frac{x_{i}}{R T} \partial_{x \alpha} \mu_{i}=\sum_{j=1}^{n_{i}} \frac{x_{j} N_{i \alpha}-x_{i} N_{j \alpha}}{D_{i j}},
$$

where $D_{i j}$ denotes the diffusivity between components $i$ and $j . \mu_{i}$ is the chemical potential for the compound $i . R$ is the gas constant and $T$ is the temperature. Several reports describe a lattice Boltzmann scheme for a multiphase flow with respect to the Maxwell-Stefan equation [62,64,65]. However, much attention was paid to the right-hand side of Eq. (17) owing to the importance of mass transfer force of the multiphase mixtures of an ideal gas system. Modeling of the dilute species transfer in a multiphase system implies the importance of the left-hand side of Eq. (17) [49]. Taking the chemical potential as the driving force is more crucial for the dilute species transfer other than simply taking the gradient of the molar fraction.

In terms of modeling the solute species, we assume no interaction happens between the solutes and the bulk flow due to the infinitely dilute property of the species. Considering a system with three bulk phases $(R, G, B)$ and the dilute species $s$, we have the relation that $x_{s} \ll x_{R}+x_{B}+x_{G}=1$ so that the molar flow can be expressed as $N_{i \alpha}=x_{i} C_{t} u_{i \alpha}$ in terms of the speed of compound $i$. The Maxwell-Stefan equation for dilute species for an incompressible and immiscible threephase system can read as

$$
-D_{s}\left(x_{R}, x_{B}, x_{G}\right) \frac{C_{s}}{R T} \partial_{x \alpha} \mu_{s}\left(x_{R}, x_{B}, x_{G}\right)=N_{\alpha}-C_{s} U_{\alpha},
$$

where

$$
\frac{1}{D_{s}\left(x_{R}, x_{B}, x_{G}\right)}=\frac{x_{R}}{D_{s R}}+\frac{x_{B}}{D_{s B}}+\frac{x_{G}}{D_{s G}} .
$$

In the above equation, $U_{\alpha}$ is the system velocity of the bulk phase according to the assumption of incompressible and immiscible system. Unlike a single-phase convection and transport equation, the distribution of the bulk phases is another factor of chemical potential so we mark the format as $\mu_{s}\left(x_{R}, x_{B}, x_{G}\right)$. The chemical potential is stated as $\mu_{s}=$ $\mu_{s}^{0}+R T \ln \left(a_{s}\right)$, where $\mu_{s}^{0}$ is the standard chemical potential of dilute species and $a_{s}$ is the chemical activity. The activity coefficient $\gamma_{s}\left(x_{R}, x_{B}, x_{G}\right)=a_{s} / x_{s}$ describes the influence of the distribution of the bulk phases. Keeping in mind that the degree of freedom is 2 due to the restriction relationship of $x_{R}+x_{B}+x_{G}=1$, we can express this coefficient by only two selected fractions of bulk phase, i.e., $\gamma_{s}\left(x_{k}, x_{l}\right)(k, l=$ $R, G, B k \neq l)$.

For the sake of analytical descriptions in the solute species in the field of the multiphase interface, we are looking for numerical approaches. A commonly used approach called twoscalar method computes the solute species with convectiondiffusion equation on each side of interface, taking a Henry coefficient as the boundary condition at the interface. The discontinuous physical property leads to higher complexity with instability of higher Henry coefficient and computational load for tracking the moving interface. From the aspect of the transition from one phase to the other, the theoretical description of the multiphase fluid mechanics named diffuse interface theory was proposed by Cahn and Hilliard in 1958 $[66,67]$. Based on the Landau and Ginzburg theory, the phase boundary is smoothly changed with a gradual mixing at the interface. The thermodynamic properties and the interface profile are derived from the free-energy function as a function of the molar fraction $x_{k}$. In a flat one-dimensional case close to the critical temperature $T_{c}$, the analytical expressions of the interface thickness are given as

$$
\begin{gathered}
x_{k}=\frac{1}{2}[1-\tanh (x / \xi)], \\
\frac{\xi}{\lambda_{0}}=2 \sqrt{\frac{T_{c}}{T_{c}-T}},
\end{gathered}
$$

where $x$ is the distance from the interface, and $\lambda_{0}$ represents a mean range of interactions [66] (i.e., in a Lennard-Jones interaction model, $\lambda_{0}$ is related to the Lennard-Jones equilibrium radius $r_{0}$ as $\lambda_{0}=r_{0} \sqrt{11 / 7}$ ). It is attainable to make a description of the interface profile knowing as the critical temperature. Therefore, we can derive the gradient of bulk phase as a function of the interface gradient based on Eq. (20) with the help of chain rule:

$$
\partial_{x \alpha} x_{k}=-\frac{2}{\xi} n_{k \alpha} x_{k}\left(1-x_{k}\right) n_{k \alpha},
$$

where $n_{k \alpha}$ is the normal vector of phase $k$, pointing out of the interface of phase $k$. Based on Eq. (22), one can get the total differentiation of each phase $k$ as shown in Eq. (23). The total differentiation is multiplied by $1 / 2$ because each interface is calculated twice in the expression of the interface vector of each phase $k$ :

$$
\begin{aligned}
\partial_{x \alpha} \ln \left(\gamma_{s}\left(x_{f}, x_{g}, x_{h}\right)\right) & =\frac{1}{2} \sum_{k=R, B, G}\left[\partial_{x_{k} \alpha} \ln \left(\gamma_{s}\right) \partial_{x \alpha} x_{k}\right] \\
& =\sum_{k=R, B, G}\left[-\frac{1}{\xi} x_{k}\left(1-x_{k}\right) \partial_{x_{k} \alpha} \ln \left(\gamma_{s}\right) n_{k \alpha}\right] .
\end{aligned}
$$

Further, the Maxwell-Stefan equation for a dilute species transfer in a three-phase system under isothermal isobaric conditions can read as follows:

$$
\begin{aligned}
N_{\alpha}= & C_{s} U_{\alpha}-D_{s}\left[\partial_{x \alpha} C_{s}\right. \\
& \left.-\frac{C_{s}}{\xi} \sum_{k=R, B, G}\left(x_{k}\left(1-x_{k}\right) \partial_{x_{k} \alpha} \ln \left(\gamma_{s}\right) n_{k \alpha}\right)\right] .
\end{aligned}
$$

At the thermodynamic equilibrium, the chemical potential $\mu_{s}$ must be equal to the equilibrium chemical potential $\mu_{s}^{0}$ so that the equilibrium state for the highly dilute compounds in a multiphase system is $\gamma_{s}\left(x_{R}, x_{B}, x_{G}\right) C_{s} / C_{t}=\exp \left[\left(\mu_{s}^{e q}-\right.\right.$ $\left.\left.\mu_{s}^{0}\right) / R T\right]$. Therefore, the interface profile can be adjusted to form the function of $\gamma_{s}\left(x_{R}, x_{B}, x_{G}\right)$ and the equilibrium state is related to the activity coefficient. From all the above, we get 
the governing equation of modeling the interface profile and the state equation of solvent solutes in a three-phase system.

The LB approach to model a highly dilute solute associates a particle distribution function $d_{i, s}$ with the discretized direction $e_{i \alpha}$ as Eq. (25). Since the mass fraction of the highly dilute is entirely small and the momentum exchange of the solute and solvents is restricted by the highly dilute assumption, the macroscopic motion can be truncated to the bulk phase, so that the equilibrium velocity and local velocity can get exclusively from the bulk phase. The governing equation of solute compound maintaining the collision and the streaming has not changed with regard to the LBM scheme type [68]:

$d_{i, s}\left(x_{\alpha}+e_{i \alpha} \delta t, t+\delta t\right)-d_{i, s}\left(x_{\alpha}, t\right)=\Omega_{s, 1}^{i}+\Omega_{s, 2}^{i}+\delta t w_{i} R_{s}$,

where $\Omega_{d, 1}^{i}$ is a single-phase collision part for the single phase, and BGK approximation is used as Eq. (26). A multiphase collision part $\Omega_{s, 2}^{i}$ is introduced to modify the interface profile and the equation of state in terms of Eq. (30). Without the statement of the collision part $\Omega_{s, 2}^{i}$, this model has been approved to recover the diffusion-convection equation with the chemical reaction part [69].

$$
\begin{gathered}
\Omega_{s, 1}^{i}=-\frac{1}{\overline{\tau_{s}}}\left(d_{i, s}\left(x_{\alpha}, t\right)-d_{i, s}^{\mathrm{eq}}\left(x_{\alpha}, t\right)\right), \\
\overline{\tau_{s}}=3 / \sum_{k} \frac{x_{k}}{D_{s, k}}+\frac{1}{2} \\
d_{i, s}^{\mathrm{eq}}\left(x_{\alpha}, t\right)=w_{i} C_{s}\left(1+\frac{e_{i \alpha} U_{\alpha}}{c_{s}^{2}}+\frac{\left(e_{i \alpha} U_{\alpha}\right)^{2}}{2 c_{s}^{4}}-\frac{U_{\alpha} U_{\alpha}}{2 c_{s}^{2}}\right), \\
C_{s}=\sum_{i=1}^{q} d_{i, s} .
\end{gathered}
$$

In the above equations, the relaxation time $\overline{\tau_{s}}$ is related to a harmonic average of the diffusivity $D_{s, k}$ with $x_{k}$ being the compound fraction in Eq. (19). It recovers the relation between the relaxation time and the diffusivity as $\tau_{s, k}=3 D_{s, k}+1 / 2$ in each single phase through the Chapman-Enskog analysis (see Appendix). $d_{i, s}^{\mathrm{eq}}$ is the corresponding equilibrium distribution for the solute shown in Eq. (28), where the equilibrium velocity $U_{\alpha}$ borrowed from the bulk phase is truncated to the second order. The discrete speed $e_{i \alpha}$ and the weight $w_{i}$ for each lattice direction are the same as the D2Q9 scheme for the bulk phase unitarily.

There are two challenges for solute modeling in terms of a multiphase system. First, the physical law for mass transfer across fluid interfaces is still not clear. Although several methods, such as Lewis and Whitman's stagnant film theory [70], Higbie's penetration theory [71], and Danckwerts's surface renewal theory [72], have contributed some basic understanding of this phenomenon, recovering the correct macroscopic behavior should be built from the microdynamic relation with a clear connection between the flexible parameters and the physical condition such as diffusivity and phase equilibrium coefficient. Second, the numerical method needs to maintain the efficiency and stability for wide-ranging parameter settings of multitudinous applications. With the further development of the LBM method, a collision operator is developed to express the solute-solvent interaction and this
TABLE II. The interfacial function and its equation of state.

\begin{tabular}{lccc}
\hline \hline Type & One phase & Partition & Interface adsorbed \\
\hline$W_{s}\left(x_{k}, 1-x_{k}\right)$ & $x_{k}-1$ & $x_{k}\left(x_{k}-1\right)$ & $x_{k}-0.5$ \\
$C_{s} / C_{\max }$ & $\left(x_{k}\right)^{-\lambda_{s, k l}}$ & $e^{\lambda_{s, k l}\left(x_{k}-1\right)}$ & $\left(4 x_{k}\left(1-x_{k}\right)\right)^{\lambda_{s, k l} / 2}$ \\
$x_{1}\left(C_{s}^{\max }\right)$ & 1 & 1 & 0.5 \\
\hline \hline
\end{tabular}

method is extended to multiphase flow,

$$
\Omega_{s, 2}^{i}=\sum_{k l k \neq l} \beta_{s, k l} W_{s}\left(x_{k}\right) d_{i, s}^{(\mathrm{eq}, 0)} n_{k l},
$$

where $d_{i, s}^{(\mathrm{eq}, 0)}=w_{i} C_{s}$ is the local equilibrium concentration with zero velocity, and the $n_{k l}=e_{i \alpha} F_{k l \alpha} /\left\|e_{i \alpha}\right\|\left\|F_{k l \alpha}\right\|$ is the normal vector of interface $k l$. In this equation, this operator is a recoloring-operator-based scheme in Eq. (30). However, the solute redistribution has a clear physical meaning compared to Eq. (24) and the activity coefficient is correlated with the interfacial profile function $W_{s}\left(x_{k}\right)$. We proceed with the Chapman-Enskog analysis of this microdynamic equation to the macroscopic convection-diffusion equation with the interface profile as (the derivation is seen in the Appendix)

$$
\begin{aligned}
\partial_{t} C_{s} & +\partial_{x \alpha}\left[C_{s} U_{\alpha}-D_{s} \partial_{x \alpha} C_{s}\right. \\
& \left.+\sum_{k l} 2 k C_{s} \tau_{s, k l} \beta_{s, k l} W_{s}\left(x_{k}\right) F_{\alpha} /\left\|F_{\alpha}\right\|\right]-R_{s}=0
\end{aligned}
$$

This equation is recovered under the limit of low Mach number with near equilibrium state and the model is accurate for the reaction part with $R_{s} \ll 1$. It shows that the equation degenerates into a convection-diffusion-reaction in a single phase flow without interface collision operator. The interface collision operator modifies the interface profile in terms of the function $W_{s}\left(x_{k}\right)$ corresponding to an activity coefficient of solute, so that the species flux can be given as

$$
\begin{aligned}
N_{\alpha}= & C_{s} U_{\alpha}-D_{s} \partial_{x \alpha} C_{s} \\
& +\sum_{k l k \neq l} 2 k C_{s} \overline{\tau_{s}} \beta_{s, k l} W_{s}\left(x_{k}\right) F_{k l \alpha} /\left\|F_{k l \alpha}\right\| .
\end{aligned}
$$

In our previous two-phase flow studies [49], the relation $\beta_{s, k l}=6 \lambda_{s, k l} \beta_{k l} \overline{D_{s}} / \overline{\tau_{s}}$ is valid for the multiphase LBM based on Latva-Kokko's work related to the interface thickness [73]. Also the parameter setting can be obtained when the interface thickness is determined by the free-energy model or other models. The interfacial function $W_{s}\left(x_{k}\right)$ related to the activity coefficient of solute in the solvents is given by Table II. The distribution of the dilute species in the interface is specially demonstrated.

When expanding this model to a ternary system, there are three typical cases of mass transfer of the solute compound. Case 1: the species soluble in one phase or adsorbed on single-interface; Case 2: the species soluble in two phases with a partition coefficient; Case 3: the species soluble in three phases with partition coefficients. The interface collision operator should be modified according to the solute properties case by case. Gathering the interface profile for different kinds of interface $k l$ in terms of the basic equation, Eq. (24), we can 
TABLE III. The operator selection and the resulting equation of state.

\begin{tabular}{|c|c|c|c|c|}
\hline \multirow{2}{*}{$\begin{array}{l}\text { Type } \\
\text { Subtype }\end{array}$} & $\begin{array}{l}\text { The compound soluble in one } \\
\text { phase or adsorbed on } \\
\text { single-interface (Only } \\
\text { dissolution in Red phase for } \\
\text { example) }\end{array}$ & \multirow{2}{*}{\multicolumn{2}{|c|}{$\begin{array}{l}\text { The compound soluble in two } \\
\text { phases with a distribution } \\
\text { coefficient (Dissolution in } \\
\text { Red \& Green phases for } \\
\text { example) }\end{array}$}} & \multirow[t]{2}{*}{$\begin{array}{c}\text { The compound soluble in } \\
\text { three phases with } \\
\text { partition coefficients }\end{array}$} \\
\hline & soluble in single-phase adsorbed on single-interface & & & \\
\hline Collision operator $\Omega_{d, 2}^{i}$ & $\beta_{s, R} W_{s}\left(x_{R}\right) d_{i, s}^{(\mathrm{eq}, 0)} n_{R \alpha}$ & \multicolumn{2}{|c|}{$\beta_{s, B} W_{s}\left(x_{B}\right) n_{B \alpha}+\beta_{s, R G} W_{s}\left(x_{R}\right) n_{R G \alpha}$} & $\sum_{k l k \neq l} \beta_{s, k l} W_{s}\left(x_{k}, x_{l}\right) d_{i, s}^{(\mathrm{eq}, 0)} n_{k l o}$ \\
\hline Interface $k l$ & Interface $R-B \& R-G$ & Interface $R-B, G-B$ & Interface $R-G$ & Interface $k-l$ \\
\hline$W_{s}\left(x_{k}\right)$ & $W_{s}\left(x_{R}\right)=x_{R}-0.5$ & $W_{s}\left(x_{B}\right)=x_{B}$ & $W_{s}\left(x_{R}\right)=-x_{R} x_{G}$ & $W_{s}\left(x_{k}, x_{l}\right)=-x_{k} x_{l}$ \\
\hline$\lambda_{s, k l}$ & $\lambda_{s, R}=\frac{\overline{\tau_{s}} \beta_{s, R}}{6 \overline{D_{s}} \beta_{k l}}$ & $\lambda_{s, B}=\frac{\overline{\tau_{s}} \beta_{s, B}}{6 \beta_{k l} \bar{D}_{s}}$ & $\lambda_{s, R G}=\frac{\overline{\tau_{s}} \beta_{s, R B}}{6 \beta_{k l} \overline{D_{s}}}$ & $\lambda_{s, k l}=\frac{\overline{\tau_{s}} \beta_{s, k l}}{6 \beta_{k l} \bar{D}_{s}}$ \\
\hline$C_{s} / C_{\max }$ & {$\left[4 x_{R}\left(1-x_{R}\right)\right]^{\lambda_{R} / 2}$} & $\left(1-x_{B}\right)^{-\lambda_{s, B}}$ & $e^{\lambda_{s, R G} x_{G}}$ & $e^{\lambda_{s, k l} x_{k}}$ \\
\hline
\end{tabular}

calculate the multiphase dynamics of the three-phase system. Table III gives the nonexhaustive overview of the possible interface collision operator of each case with regard to Eq. (32). The next part gives a derivation under each case.

\section{The compound soluble in one phase or adsorbed on single interface}

For the sake of clearness in the explanation, we assume that the solute compound only dissolves in Red phase in this subsection. The distributions in the Blue and Green phases have no influence on the dynamic behavior of the solute compound, making it possible to read the activity coefficient $\gamma_{s}\left(x_{R}\right)$ only as a function of the compound fraction $x_{R}$. The interface $R-B$ and $R-G$ can be taken into consideration together with the assumption of the same interface thickness, so the interface vector can be read as

$$
n_{R \alpha}=\frac{F_{R B \alpha}+F_{R G \alpha}}{\left\|F_{R B \alpha}+F_{R G \alpha}\right\|} .
$$

Therefore, the governing equation, Eq. (24), degenerates into

$$
N_{\alpha}=C_{s} U_{\alpha}-D_{s} \partial_{x \alpha} C_{s}+\frac{2 D_{s} C_{s}}{\xi} x_{R}\left(1-x_{R}\right) \partial_{x_{R} \alpha} \ln \left(\gamma_{s}\right) n_{R \alpha} .
$$

Compared with the macroscopic equation, Eq. (32), derived from the LB model Eq. (A18), the activity coefficient can be expressed as a linear local equation:

$$
\partial_{x_{R} \alpha} \ln \left(\gamma_{s}\right)=\frac{\overline{\tau_{s}} \beta_{s, R} k \xi}{D_{s}} \frac{W_{s}\left(x_{R}\right)}{x_{R}\left(1-x_{R}\right)} .
$$

In this study, we set $\beta_{k l}=0.7$ as the same as that in Latva-Kokko's work [73], where the interface thickness of about 6-8 1.u. (lattice units) is able to maintain the numerical accuracy of interface behavior. As we proposed before, the part of $k \xi$ reflecting the interface thickness can be replaced by $1 / 6 \beta_{k l}$, resulting from modifying the interface thickness from the color-gradient model [49]. We define the partition parameter $\lambda_{s, R}=\overline{\tau_{s}} \beta_{s, R} / 6 \beta D_{s}$ under the circumstance that the solute is only soluble in the Red phase. We can obtain the single-phase soluble compound and single-interface adsorbed compound with the possible choice of the interface function $W_{s}$ in Table III.

\section{The compound soluble in two phases with a partition coefficient}

This part solves the condition that dilute solute is soluble in two phases with a partition coefficient without being soluble in another phase. For the sake of concreteness and simplicity without losing generality, the compound is soluble in Red and Green phases with a partition coefficient. Note that the governing equation is expanded to a function of normal vector $n_{k \alpha}$ in Eq. (24). In order to derive the interface profile, the normal vector can be expanded with the help of the color-gradient force as a function of the normal vector of each interface $n_{k l \alpha}$ :

$$
n_{k \alpha}=\frac{\left\|F_{k l \alpha}\right\|}{\left\|F_{k l \alpha}+F_{k m \alpha}\right\|} n_{k l \alpha}+\frac{\left\|F_{k m \alpha}\right\|}{\left\|F_{k l \alpha}+F_{k m \alpha}\right\|} n_{k m \alpha},
$$

with the help of the interface vector relation:

$$
n_{k l \alpha}=-n_{k l \alpha},
$$

Eq. (24) can be reformed as

$$
\begin{aligned}
N_{\alpha}= & C_{s} U_{\alpha}-D_{s} \partial_{x \alpha} C_{s} . \\
& +\frac{D_{s} C_{s}}{\xi} \sum_{k l k \neq l \neq m}\left(x_{k}\left(1-x_{k}\right) \partial_{x_{k} \alpha} \ln \left(\gamma_{s}\right) \frac{\left\|F_{k l \alpha}\right\|}{\left\|F_{k l \alpha}+F_{k m \alpha}\right\|}\right. \\
& \left.-x_{l}\left(1-x_{l}\right) \partial_{x_{l} \alpha} \ln \left(\gamma_{s}\right) \frac{\left\|F_{k l \alpha}\right\|}{\left\|F_{l m \alpha}+F_{k l \alpha}\right\|}\right) n_{k l \alpha}
\end{aligned}
$$

Specially, the governing equation for this case can be changed to

$$
\begin{aligned}
N_{\alpha}= & C_{s} U_{\alpha}-D_{s} \partial_{x \alpha} C_{s} \\
& +\frac{D_{s} C_{s}}{\xi}\left[2 x_{B}\left(1-x_{B}\right) \partial_{x_{B} \alpha} \ln \left(\gamma_{s}\right) n_{B \alpha}\right. \\
& +\left(x_{R}\left(1-x_{R}\right) \partial_{x_{R} \alpha} \ln \left(\gamma_{s}\right) \frac{\left\|F_{R G \alpha}\right\|}{\left\|F_{R G \alpha}+F_{R B \alpha}\right\|}\right. \\
& \left.\left.-x_{G}\left(1-x_{G}\right) \partial_{x_{G} \alpha} \ln \left(\gamma_{s}\right) \frac{\left\|F_{G R \alpha}\right\|}{\left\|F_{G R \alpha}+F_{G B \alpha}\right\|}\right) n_{R G \alpha}\right] .
\end{aligned}
$$

Note that the compound fraction $x_{B} \rightarrow 0$ at the $R-G$ interface, and we have a weak relation $x_{G} \approx 1-x_{R}$. Based on this assumption, we can read Eq. (39) as

$$
\begin{aligned}
N_{\alpha}= & C_{s} U_{\alpha}-D_{s} \partial_{x \alpha} C_{s}+\frac{D_{s} C_{s}}{\xi}\left[2 x_{B}\left(1-x_{B}\right) \partial_{x_{B} \alpha} \ln \left(\gamma_{s}\right) n_{B \alpha}\right. \\
& \left.+2 x_{R} x_{G} \partial_{x_{R} \alpha} \ln \left(\gamma_{s}\right) n_{R G \alpha}\right] .
\end{aligned}
$$


In order to make a comparison between Eq. (24) and Eq. (40), a straightforward identification of $n_{B \alpha}$ and $n_{R G \alpha}$ of the interface operator forms as a combination of interface collision operator in Table II:

$$
\Omega_{s, 2}^{i}=\beta_{s, B} W_{s}\left(x_{B}\right) n_{B \alpha}+\beta_{s, R G} W_{s}\left(x_{R}\right) n_{R G \alpha} .
$$

In the above specific equation, the interface profile is explicated with an interface function $W_{s}\left(x_{k}\right)$. Similar to Eq. (35), the interface function is modified through the comparison between the collision operator and the interface profile separately. The straightforward comparison between the Henry coefficient and the parameter $\lambda_{s, R G}$ as

$$
H_{R G}=\frac{C_{s, R}^{\mathrm{eq}}}{C_{s, G}^{\mathrm{eq}}}=e^{\lambda_{s, R G}}
$$

\section{The compound soluble in three phases with partition activity coefficients}

This part solves the condition that dilute solute is soluble in the three-phase system with partition coefficients. Before going further to modify the collision operator, the basic equation Eq. (24) can be organized as a function of the normal vector $n_{k l \alpha}$ of interface $k l$ instead of the normal vector $n_{k \alpha}$ :

$$
N_{\alpha}=C_{s} U_{\alpha}-D_{s} \partial_{x \alpha} C_{s}+\frac{2 D_{s} C_{s}}{\xi} \sum_{k l k \neq l} x_{k} x_{l} \partial_{x_{k} \alpha} \ln \left(\gamma_{s}\right) n_{k l \alpha}
$$

Similar to Sec. II B1, through the comparison between Eqs. (32) and (44), a straightforward identification of the interface profile is given as the following:

$$
\Omega_{s, 2}^{i}=\sum_{k l k \neq l} \beta_{s, k l} W_{s}\left(x_{k}, x_{l}\right) d_{i, s}^{(\mathrm{eq}, 0)} n_{k l \alpha} .
$$

The interface function $W_{s}\left(x_{k}, x_{l}\right)$ is related to partition activity coefficient of the solute at the interface $k l$. With the help of the deviation shown in Table II, Table III gives the choice of the applications of the $W_{s}\left(x_{k}, x_{l}\right)$ of interface $k l$. The comparison between the action of $\lambda_{s, k l}$ and the analytical solution of the partition coefficient can be found as

$$
\begin{aligned}
& \frac{C_{s, R}^{\mathrm{eq}}}{C_{s, B}^{\mathrm{eq}}}=H_{R B}=e^{\lambda_{s, R B}}, \\
& \frac{C_{s, G}^{\mathrm{eq}}}{C_{s, B}^{\mathrm{eq}}}=H_{G B}=e^{\lambda_{s, G B}}, \\
& \frac{C_{s, R}^{\mathrm{eq}}}{C_{s, G}^{\mathrm{eq}}}=\frac{H_{R B}}{H_{G B}}=H_{R G}=e^{\lambda_{s, R B}-\lambda_{s, G B}}=e^{\lambda_{s, R G}},
\end{aligned}
$$

where the derivation of the Henry coefficient has clear definition in terms of $\lambda_{s, k l}$ at each interface. According to Eq. (46), $\lambda_{s, k l}$ is the key parameter to control the partial soluble dilute species and one of the three parameters is determined by the other two. The relation of the $\lambda_{s, k l}$ can be derived from the relation of the Henry coefficient as Eq. (46):

$$
\lambda_{s, R B}-\lambda_{s, G B}=\lambda_{s, R G} .
$$

It is noted that the changes of chemical potential $\mu_{s}$, or the activity $a_{s}$ reflect the variation of enthalpy and entropy of the solvent composition so that this model should evolve not far away from the equilibrium state. A wide span of possible applications can be modeled since the properties of microfluidic system and the microfluidic interface evolve close to the equilibrium. Also, the interfacial function $W_{s}\left(x_{B}, x_{R}, x_{G}\right)$ can be redesigned according to the equation of equilibrium in the three-phase system.

\section{NUMERICAL RESULTS AND DISCUSSIONS}

In the following section, we will illustrate the properties of the proposed model through numerical tests. The bulk phase model uses a ternary color-gradient model described above in Ref. [31] with an identical interface thickness of $\beta_{k l}=$ 0.7 based on D2Q9 scheme. The interface function of $W_{s}$, the partition coefficient $\lambda_{s}$, and the diffusion parameter $\bar{\tau}_{s}$ of the transport equation were well discussed in two-phase model in our previous work [49]. Here we start from stability analysis by a ternary phase decomposition test. Next parts are provided for the illustration and validation of the selection of partition coefficient $\lambda_{s, k l}$, including the parameter setting of $\lambda_{s, k l}$, detailed comparison with Higbie's penetration theory and mass transport in a liquid lens. An application of this model to simulate interphase mass transfer in Janus droplet is further investigated.

\section{A. Model convergence in the phase decomposition}

Phase decomposition, usually called spinodal decomposition, can be considered as a physical process of separation of a heterogeneous or homogeneous mixture. Here, we focus on the convergence of the dilute solute model in the process of phase decomposition of a random multiphase mixture. This chaotic distribution of the initial condition is a convergence toward a stable equilibrium state due to tiny concentration fluctuation at interface, which can be an excellent numerical case to test the stability of the multiphase solute transfer model. Although this study of the solute compound is limited by physical meaning, these cases make it possible to validate the robustness of the model and the final state of the solute aggregation. These numerical cases are devoted to the heterogeneous mixture separation, and the computational domain is a $N_{x} \times N_{y}=$ $120 \times 120$ lattice sites with periodic boundary conditions. Meanwhile, the colors of three fluids (Red, Green, Blue) are selected in each site with a uniform probability and then the local distribution function is initialized by the zero velocity equilibrium of the color fluid. The physical parameters of bulk phase are fixed: the interface tension $\gamma_{B R}=\gamma_{B G}=\gamma_{R G}=$ 0.01 , the kinematic viscosity $v_{B}=v_{R}=v_{G}=1 / 6$, and the density $\rho_{B}=\rho_{R}=\rho_{G}=1$. The robustness and the stability of bulk phase model are given by Ref. [31].

The dilute compound is added into the system with the same distribution of Red phase as the initial condition. The collision operators are selected to validate the numerical stability of these four different compounds listed in Table III, and corresponding parameters are given in Table IV. A wide range of the relaxation time, the partition coefficient $\lambda_{s, k l}$, and the interface function $W_{s}\left(x_{k}, x_{l}\right)$ are examined. The temporal evolutions of the convergence with $\lambda_{s, k l}=1$ are shown in Figs. 2-5, respectively. Note that color-gradient model is a 
TABLE IV. The selected parameter functions and the resulting equation of state.

\begin{tabular}{lccc}
\hline \hline & Case 1 & Case 2 & Case 3 \\
\hline$\Omega_{s, 2}^{i}$ & $\beta_{s, R} W_{s}\left(x_{R}\right) d_{i, s}^{(\mathrm{eq}, 0)} n_{R \alpha}$ & $\beta_{s, B} W_{s}\left(x_{B}\right) d_{i, s}^{(\mathrm{eq}, 0)} n_{B \alpha}+$ & $\sum_{k l k \neq l} \beta_{s, k l} W_{s}\left(x_{k}, x_{l}\right) d_{i, s}^{(\mathrm{eq}, 0)} n_{k l \alpha}$ \\
$W_{s}$ & $W_{s}\left(x_{R}\right)=x_{R}-1 W_{s}\left(x_{R}\right)=x_{R}-0.5$ & $\beta_{s, R G} W_{s}\left(x_{R}\right) d_{i, s}^{(\mathrm{eq}, 0)} n_{R G \alpha}$ & $W_{s}\left(x_{k}, x_{l}\right)=-x_{k} x_{l}$ \\
$\tau_{s, k}$ & $\lambda_{s, R}=1$ & $W_{s}\left(x_{B}\right)=x_{B} W_{s}\left(x_{R}\right)=-x_{R} x_{G}$ & \\
$\lambda_{s}$ & $\tau_{s, k} \in\{1,0.505\}$ for each phase & \\
\hline \hline
\end{tabular}

diffuse-interface-based method so we represent the interface as the range of the density value from 0.3 to 0.7 by dash line in figures. The thickness of interface usually takes 4-6 1.u. The results show that the algorithm of dilute compound is stable under three-phase convergence with regard to different types of interface function $W_{s}\left(x_{k}, x_{l}\right)$. Besides, with the aggregation of the solvent component as time goes on, the dilute compound acts as the definition of the interface function $W_{s}$ : the dilute compound is trapped in the Red phase as Case 1; the dilute compound is adsorbed at the $R-G$ and $R-B$ interfaces as Case 2 ; the dilute compound is soluble in Red \& Green phase with a partition coefficient while the compound is insoluble in Blue phase as Case 3, where Red phase has a larger concentration at the final equilibrium as the result of the parameter setting. Case 4 shows that the dilute compound is soluble in three-phase with partition coefficients, and the equilibrium concentration has an order of $C_{s, R}>C_{S, G}>C_{S, B}$ owing to the setting of the three parameters $\lambda_{s, B R}>\lambda_{s, B G}>\lambda_{s, R G}$.

\section{B. Mass transfer in a multilayered planar interface}

The goal in this section is to demonstrate the model's capability to accurately predict the final equilibrium state of solute and the mass transfer across a fluid interface, which is tested through the multilayered planar interface benchmark with the following configuration.

The computational mesh is adopted as $N_{x} \times N_{y}=50 \times$ 180 with periodic boundary condition at the side of computational domain. The profile of the bulk phase is initialized by

$f(x, y)=\left\{\begin{array}{l}f^{B}=f^{B, \mathrm{eq}}\left(u_{\alpha}=0\right), f^{R}=f^{G}=0 \quad y \leqslant 90 \\ f^{R}=f^{R, \mathrm{eq}}\left(u_{\alpha}=0\right), f^{B}=f^{G}=0 \quad 90<y \leqslant 180 \\ f^{G}=f^{G, \mathrm{eq}}\left(u_{\alpha}=0\right), f^{B}=f^{R}=0 \quad y>180,\end{array}\right.$

where $f^{k \text {,eq }}$ is the equilibrium distribution function with the velocity equal to zero. The parameters of fluid properties are the same as in Sec. III A. Initially, solute compound is added

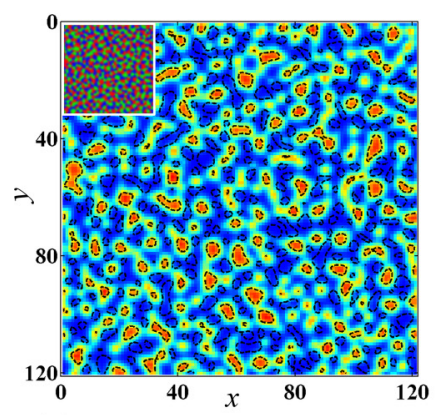

(a)

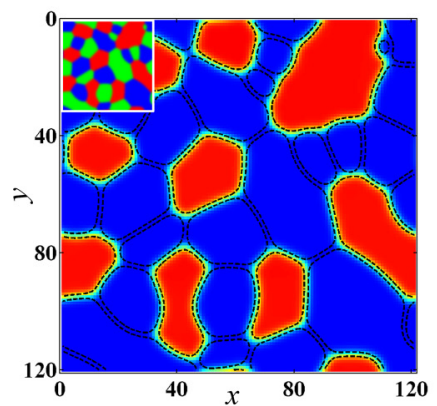

(d)

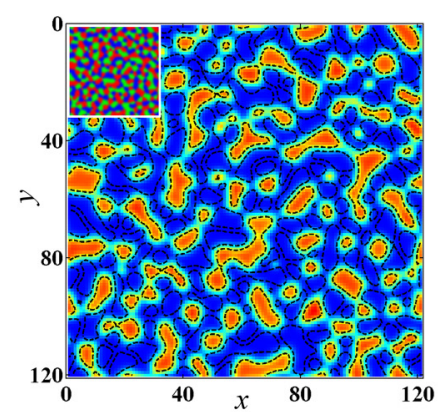

(b)

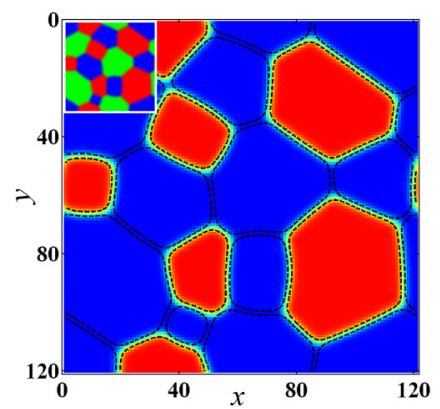

(e)

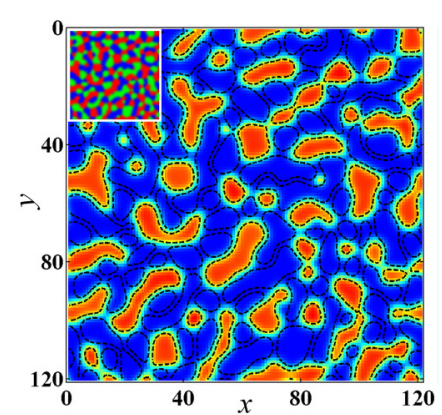

(c)

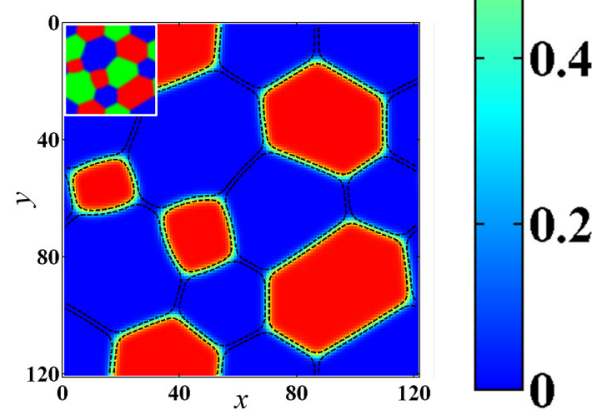

(f)

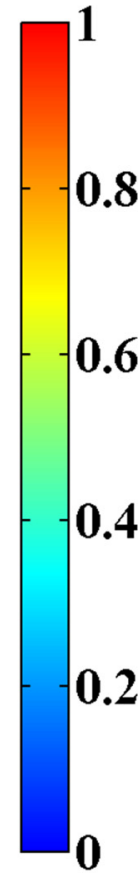

0.8

4

\section{2}

FIG. 2. The temporal evolution of the one-phase soluteble compound in Case 1 during phase segragation, where the dilute compound only stays in the Red phase. The inset shows the coresponding bulk phase flow field by a RGB image as the color defination of the fluids (The black, dark gray, and light gray represent Blue phase, Red phase, and Green phase, respectively). The coordinates of $x, y$ axes are repreneted by the number of lattices. The time step (t.s. in short) is (a) 100 t.s., (b) 500 t.s., (c) 1000 t.s., (d) 10000 t.s., (e) 50000 t.s., (f) 100000 t.s. 


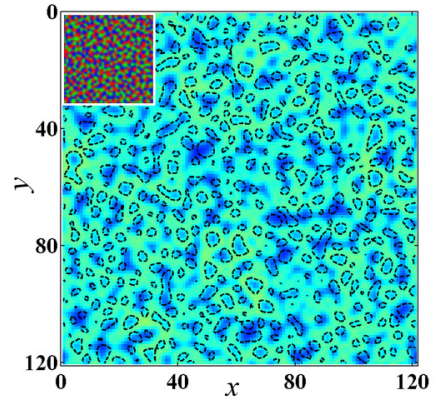

(a)

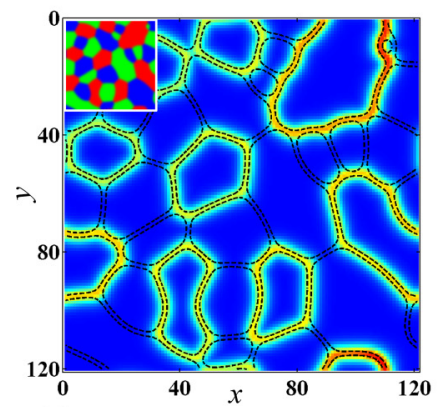

(d)

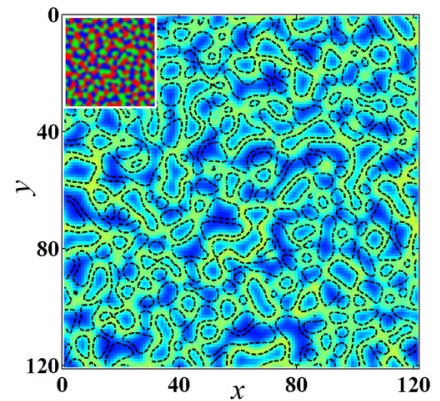

(b)

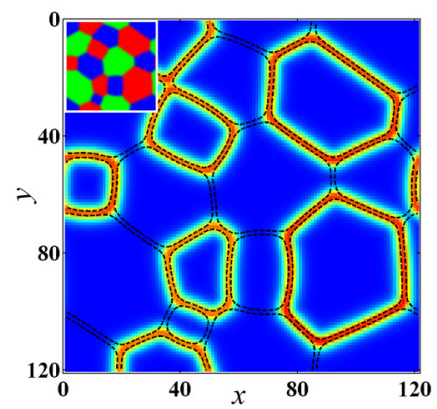

(e)

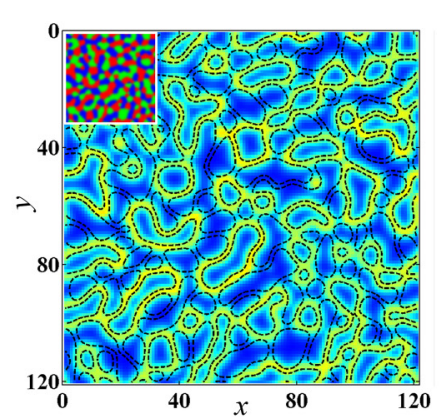

(c)

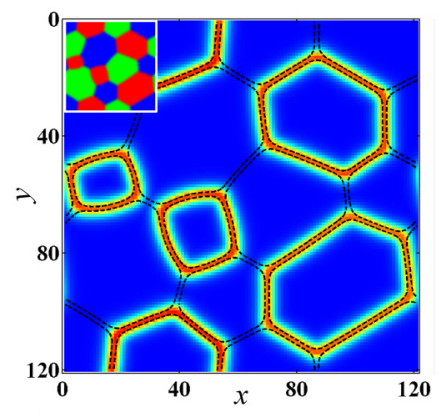

(f)

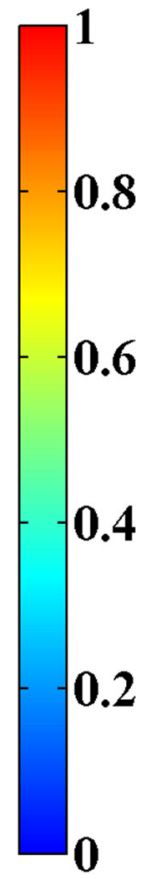

0.8
0.6

0.4

0.2

FIG. 3. The temporal evolution of adosrbed coumpound at the interface R-B and R-G in Case 2 during the phase segragation. The inset shows the coresponding bulk phase flow field by a RGB image as the color defination of the fluids (The black, dark gray, and light gray, represent Blue phase, Red phase, and Green phase, respectively). The coordinates of $x, y$ axes are repreneted by the number of lattices. The time step (t.s. in short) is (a) 100 t.s., (b) 500 t.s., (c) 1000 t.s., (d) 10000 t.s., (e) 50000 t.s., (f) 100000 t.s.

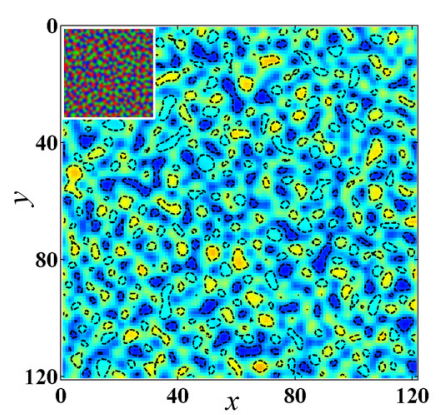

(a)

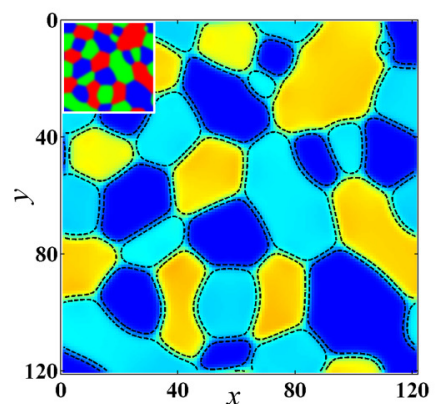

(d)

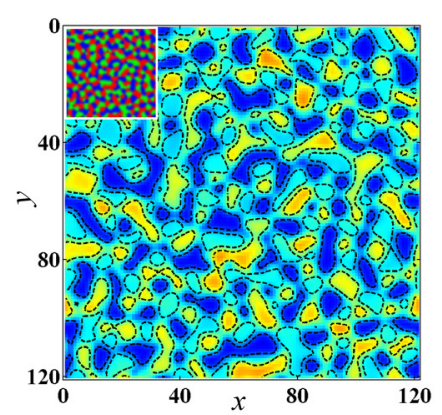

(b)

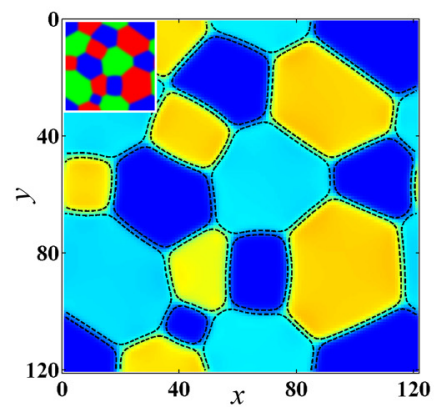

(e)

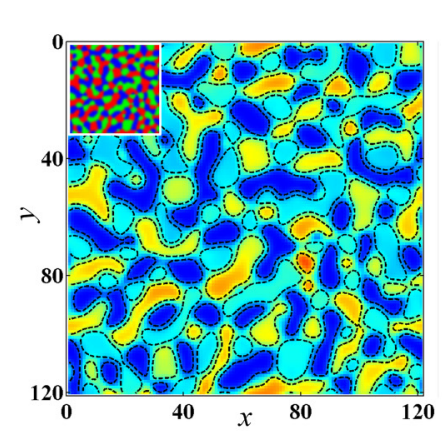

(c)

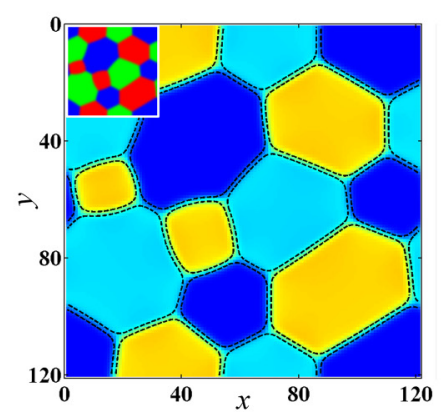

(f)

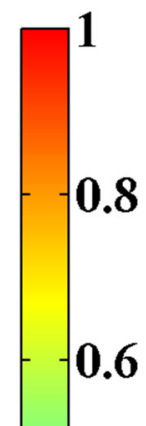

$-0.4$

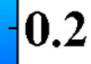

FIG. 4. The temporal evolution of the two-phase soluble compound in Case 3 during phase segragation, where the compound is more soluble in Red phase than that in Green phase. The inset shows the coresponding bulk phase flow field by a RGB image as the color defination of the fluids (The black, dark gray, and light gray represent Blue phase, Red phase, and Green phase, respectively). The coordinates of $x, y$ axes are repreneted by the number of lattices. The time step (t.s. in short) is (a) 100 t.s., (b) 500 t.s., (c) 1000 t.s., (d) 10000 t.s., (e) 50000 t.s., (f) 100000 t.s. 


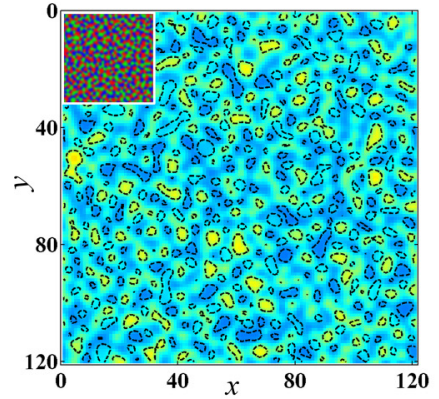

(a)

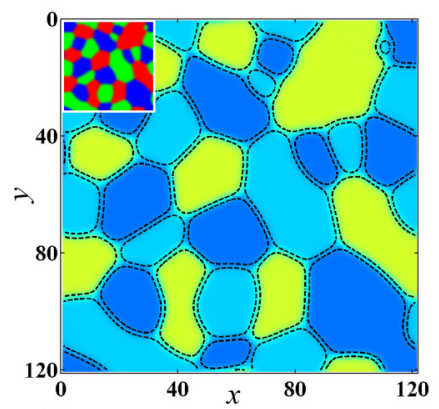

(d)

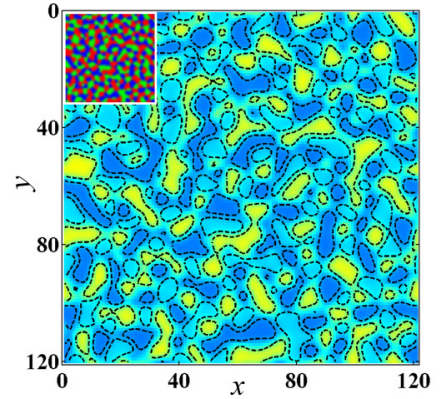

(b)

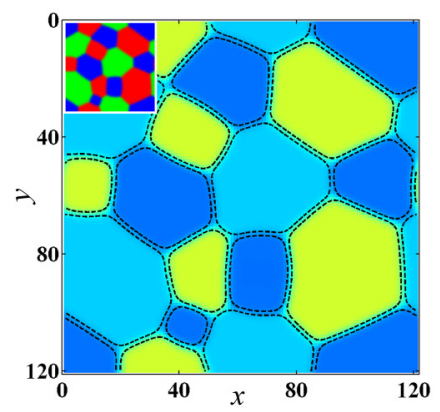

(e)

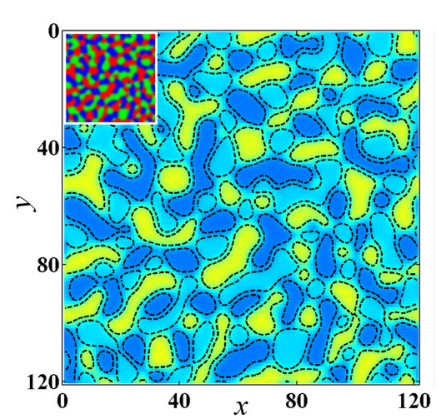

(c)

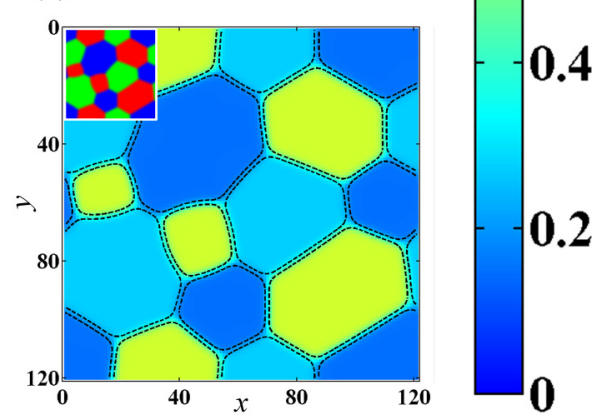

(f)

FIG. 5. The temporal evolution of the three-phase soluteble compound in Case 1 during phase segragation, where the equilibrium concentration is $C_{s, R}>C_{s, G}>C_{s, B}$. The inset shows the coresponding bulk phase flow field by a RGB image as the color defination of the fluids (The black, dark gray, and light gray represent Blue phase, Red phase, and Green phase, respectively). The coordinates of $x, y$ axes are repreneted by the number of lattices. The time step (t.s. in short) is (a) 100 t.s., (b) 500 t.s., (c) 1000 t.s., (d) 10000 t.s., (e) 50000 t.s., (f) 100000 t.s.

into the system after 10,000 t.s. steps when the convergence of the bulk phase leads to a smooth change of the interface and the mass-transfer of the solute would be completely controlled by the added collision operator. The solute compound located in Red phase has the same distribution of the Red phase and progressively diffuses across the interface R-G and R-B. The relaxation time is adopted as $\tau_{s, k} \in\{1.4,1,0.55\}$ in order to include the action of $\lambda_{s, k l}$ for generality. The phasefield dependent diffusivity can be obtained from Eq. (19). The compound crosses the interface in to Blue phase and Green phase and then reaches the equilibrium state of partial soluble in three phases. The equilibrium state is checked with $\sum_{N_{x} \times N_{y}}\left|\frac{C_{d}(t)-C_{d}(t-1)}{C_{d}(t)}\right|<10^{-5}$.

The action of $\lambda_{s, k l}$ is the key for interface topology, and the equilibrium state is related to the partition coefficient. We investigate the equilibrium state by comparing it with the analytical solution in Table III. The concentration of compound is chosen as $C_{s, R}>C_{s, G}>C_{s, B}$ without loss of generality, obtained from the definition that $\lambda_{s, B R}>\lambda_{s, R G}>$ $\lambda_{s, B G}$. Figure 6 shows the numerical results on the agreement between the action of $\lambda_{s, k l}$ and the Henry coefficient. The restriction of parameter setting of $\lambda_{s, k l}$ in Eq. (45) is examined and the largest Henry coefficient $H_{s, B G}$ up to 20 is consistent with the analytical solution given in dotted line. The deviation of the Henry coefficient between the numerical results and theoretical prediction resulting from the error part [given in Eq. (A19)] cannot be neglected when it comes to larger $\lambda_{s, k l}$. The deviation of the partition coefficients between the analytical and numerical ones increases exponentially as the parameter $\lambda_{S, B R}$ related to the largest Henry coefficient is greater than 3 (i.e., the deviation would reach up to $90 \%$ when $\lambda_{s, B R}=5, \lambda_{s, R G}=4.8, \lambda_{s, B G}=0.2$ ). The test is also conducted with a constant velocity $U=0.011$.u./t.s. toward the $x$ direction of the whole system, and the same results of the equilibrium state of the dilute solute are achieved by varying the retaliation time $\tau_{s, k}$. The equilibrium state rarely depends on the setting of the action of $\lambda_{s, k l}$ in the ternary system.

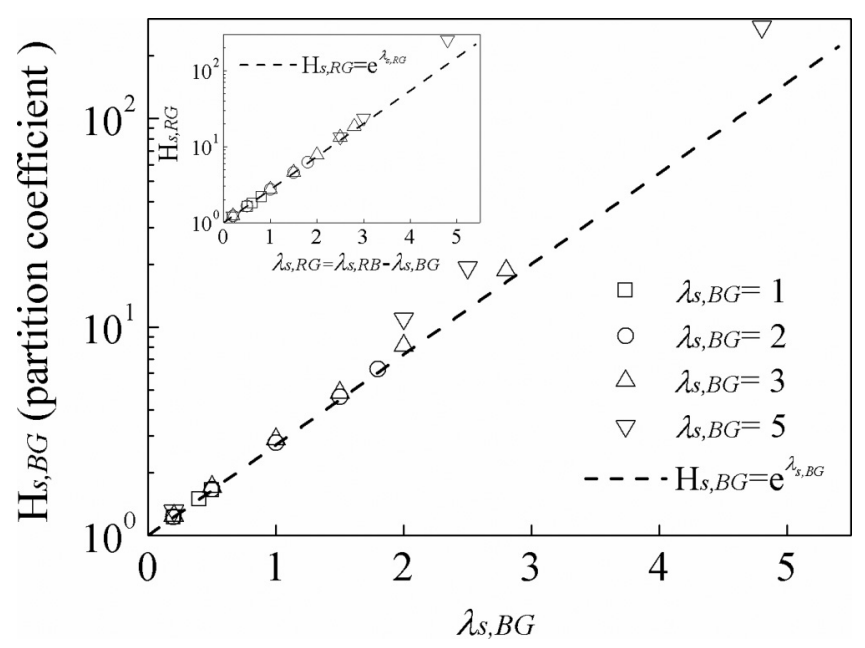

FIG. 6. The effect of $\lambda_{s, B G}, \lambda_{s, R B}$ on the Henry coefficient of the three-phase soluble compound condition. 

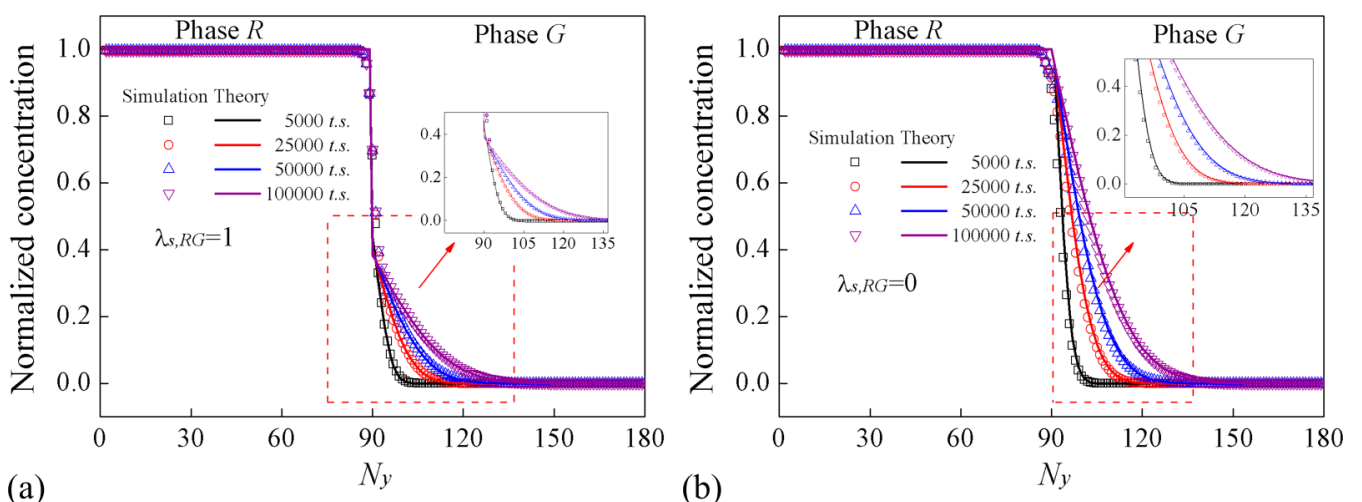

FIG. 7. The evolution of solute mass transfer across the interface compared with the penetrate theory from Eq. (48); (a) $\lambda_{s, R G}=1$; (b) $\lambda_{s, R G}=0$. The dotted lines show the numerical results at each time step (t.s.) and the solid lines show the corresponding theoretically results.

\section{Mass transfer in a multilayered planar interface}

Another concern is the interface transport phenomena. This benchmark is validated for two bulk phase models from our previous work through the comparison with numerical results from the COMSOL solver [49]. In this section, this numerical result is designed to validate the mass transfer at interface restrictively for the interfaces for the three-phase system. Very few reports have explicitly and physically dedicated to the problem of mass transfer of solute in multiphase flows owing to the complexity of the solute transport dynamics near the interface. Usually, reasonable mathematical description can be established based on some physical assumptions. Lewis and Whitman [70] in 1924 firstly proposed the double film theory for the gas-liquid system. Their assumption that the mass transfer resistance lies in two films on both sides of the interface is only applicable to a state of diffusion process and fails to predict the transfer with surface wave or complex boundary. A more general model for the unsteady diffusion process is the Penetration theory proposed by Higbie et al. [71], assuming that the solute in constant random motion arrives at the interface during a fixed period of time. The diffusion penetrating the interface is caused by a number of the molecules while the other groups of molecules stay in the original phase. A further developed theory called Surface Renewal theory improved the Penetration theory with regard to the different periods of time of arriving interface, while the rate of renewal of the surface is hard to measure experimentally [72]. Although the mass transfer behavior at interface is modified differently, these multiphase mass transfer theories all stated an equilibrium condition with Henry coefficient at the interface. In this benchmark, the numerical case is set to be compared with an analytical equation from the Penetration theory. We implement a $N_{x} \times N_{y}=122 \times 180$ computational domain with the periodic boundary along $x$ direction, and the solid wall at the upper and below are adopted the half-way bounce back scheme. The solid boundaries are set as fully wetted by phase B by setting the density of phase B in the solid nodes [50]. The parameters for the bulk phase are the same as those in Sec. IIIB. The top half is initialized with the Red phase whereas the rest is initialized with the Green phase. This multiphase flow simulation converges with a smooth interface of 6 l.u. after 10,000 t.s. In order to be compared with the analytical equation derived from the penetration theory for a certain case, it assumes the mass transfer resistance in Green phase and the boundary is far away from the interface. In order to fit this assumption, we refresh the dilute compound concentration in Red phase in each time step, and the diffusivity is rather large in Red phase. Note that the analytical model fails if the dilute species reaches the boundary phase adhere to Green phase. In the early time of mass transfer, the concentration of solute penetrates the interface into Green phase in this numerical case and obeys the following equation:

$$
C_{s}(y, t)=C_{i}\left(\lambda_{s, R G}\right) \operatorname{efrc}\left(\frac{y-N_{y} / 2}{2 \sqrt{D_{h} t}}\right)\left(y>N_{y} / 2\right),
$$

where $C_{i}\left(\lambda_{s, R G}\right)$ is the equilibrium concentration at the interface which can be derived from the partition coefficient $\lambda_{s, R G}$, meanwhile efrc is the Gauss error function. In order to keep the resistance of mass transfer in Red phase neglectable, we choose $D_{R}=7 / 30$ and $D_{G}=1 / 600$, with a diffusion ratio up to 140 . The solute is added in Red phase firstly and refreshed as the initial value in Red phase in each time step. Figure 7 shows the agreement of the analytical solution by Eq. (48) and the simulation from 5000 to 100000 t.s.. These cases verify that the solute keeps a smooth transition across the interface with equations in Table III and the thermal equilibrium assumption at the interface could be adjusted with regard to the action of $\lambda_{s, R G}$. In addition, Galilean invariance is checked as shown in Ref. [49].

To study the effect of the action of $\lambda_{s, R G}$ and relaxation time $\tau_{s, G}$ on the accuracy of our model, we compute the relative error for each combination of $\lambda_{s, R G}$ and $\tau_{s, G}$, defined as Eq. (49). Table V shows the relative error decreases as the time step increases. This is probably due to the fact that the initial condition is not accurate for each group of parameter setting. This model would become unstable at very low relaxation time or very high $\lambda_{s, R G}$, which is probably due to the nature of diffusion of the LB scheme:

$$
E_{R}=\frac{\sum_{y}\left|C_{d, \text { numerical }}(\mathbf{x}, t)-C_{d, \text { theoretical }}(\mathbf{x}, t)\right|}{\sum_{y}\left|C_{d, \text { theoretical }}(\mathbf{x}, t)\right|} .
$$


TABLE V. Relative errors between the analytical and numerical results for different $\lambda_{s, R G}$ and relaxation time $\tau_{s, G}$.

\begin{tabular}{lcccc}
\hline \hline & & \multicolumn{3}{c}{ Time steps } \\
\cline { 3 - 5 }$\lambda_{s, R G}$ & $\tau_{s, G}$ & 25000 & 50000 & 100000 \\
\hline 0 & 0.505 & $7.31 \mathrm{E}-02$ & $5.42 \mathrm{E}-02$ & $4.08 \mathrm{E}-02$ \\
0 & 0.501 & $1.02 \mathrm{E}-01$ & $6.43 \mathrm{E}-02$ & $3.42 \mathrm{E}-02$ \\
1 & 0.505 & $7.84 \mathrm{E}-02$ & $7.22 \mathrm{E}-02$ & $6.93 \mathrm{E}-02$ \\
1 & 0.501 & $8.70 \mathrm{E}-02$ & $9.01 \mathrm{E}-02$ & $9.26 \mathrm{E}-02$ \\
2 & 0.505 & $7.02 \mathrm{E}-02$ & $7.86 \mathrm{E}-02$ & $8.89 \mathrm{E}-02$ \\
\hline \hline
\end{tabular}

\section{Mass transfer in a liquid lens}

The liquid lens is a widely used benchmark to test the convergence and ability of numerical model for a three-phase flow $[7,11,31,33,36]$. This section aims to verify the model capability to predict inter-phase mass transfer at a triple fluid junction. The equilibrium state and the mass transfer at interface are examined separately in the previous sections, so this numerical test is going to check the behavior of dilute compound in a three-phase flow. A computational domain is used with periodic boundary conditions at the ends along the $x$ direction. Whereas the upper and lower boundaries use the half-way bounce back scheme. The solid boundaries are set as fully wetted by phase B. The initialization of the Red phase is as follows:

$$
\left(x-\frac{N_{x}-1}{2}\right)^{2}+\left(y-\frac{N_{y}-1}{2}\right)^{2} \leqslant R^{2},
$$

where $R$ is the initialized circular radius with the value of 30 l.u., and the rest sites in the section $y-\frac{N_{y}-1}{2}$ is initialized by the Green phase while the other rest sites are filled with Blue phase. The subject of the contact angles at the triple junction could be referred to a variety of literatures $[30,36]$. The parameters are set as $v_{B}=v_{R}=v_{G}=1 / 6, \rho_{B}=\rho_{R}=$ $\rho_{G}=1$. Especially, in order to testify the mass transfer of dilute compound, a typical case of interface tension is chosen as $\sigma_{B R}=0.014 \sigma_{B G}=0.006 \sigma_{R G}=0.01$, leading to a semicircle of Red phase locating in the center of the simulation domain. The stabilized condition of bulk phase is checked by $\sum_{N_{x} \times N_{y}}\left|\frac{\rho_{R}(t)-\rho_{R}(t-1)}{\rho_{R}(t)}\right|<10^{-5}$. The bulk phase fluid dynamics and the shape of Red phase have been well addressed in Ref. [31], where the capability for simulating flows with a high viscosity ratio and/or density ratio has been demonstrated.

The dilute compound is added into the system with the same distribution of Red phase and the action of $\lambda_{k, k l}=1$ for all interfaces. The relaxation time is set as $\tau_{d, R}=0.6, \tau_{d, G}=0.7$, making the ratio of diffusion parameter in Red phase twice of that in Green phase. In this section, the case of the two-phase soluble compound and the three-phase soluble compound are examined in the liquid lens case. Figure 8 shows the pseudocolor images of concentration of the two-phase compound at different instances of time. The counter line is added to represent the distribution of the compound clearly. The most concerned aspect is the evolution behavior of the dilute compound and the final equilibrium state in the presence of the triple fluid conjunction. The solute penetrates the interface $R-G$ whereas the solute is inhibited through interface $\mathrm{B}-\mathrm{R}$ and $\mathrm{B}-\mathrm{G}$ due to the definition of the interface profile.

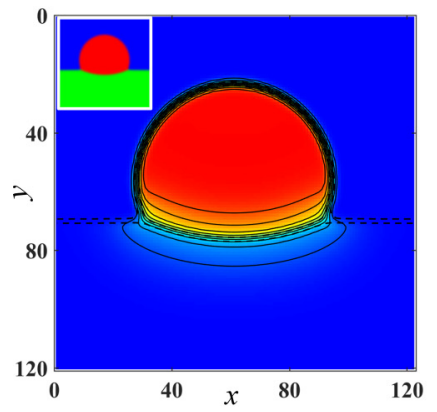

(a)

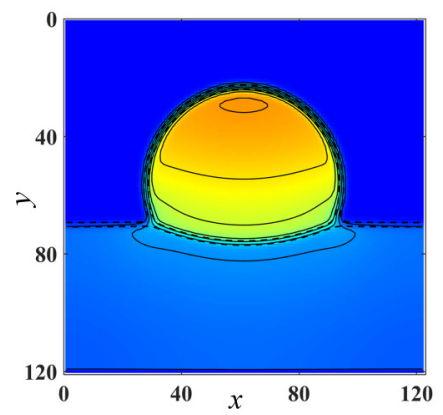

(d)

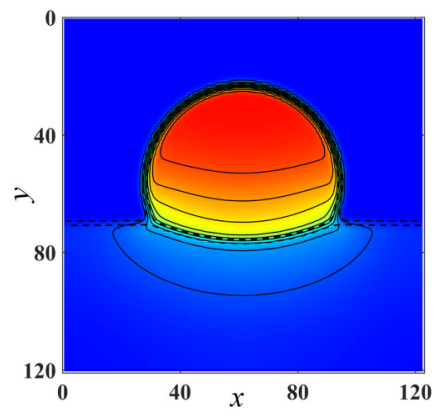

(b)

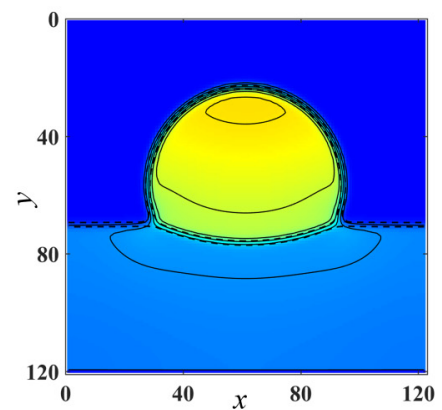

(e)

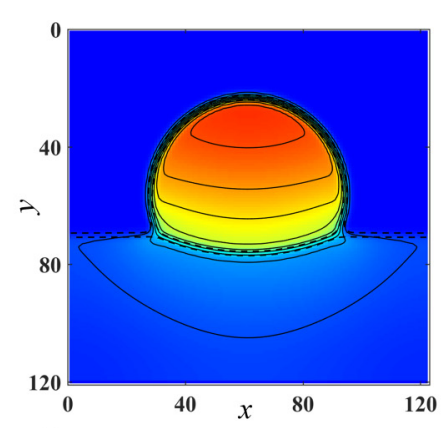

(c)
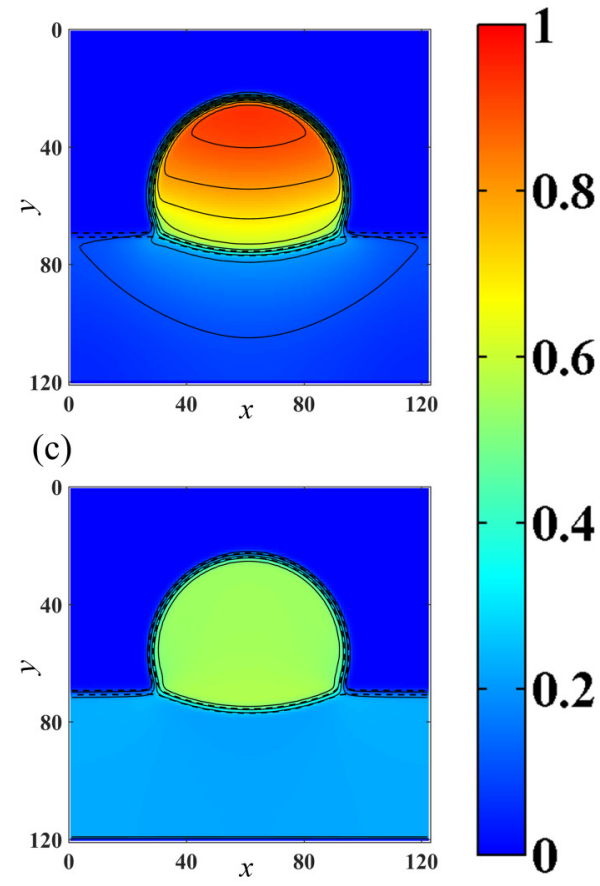

(f)

FIG. 8. The pseudocolor images representing the mass transfer of the two-phase soluble compound in the equilibrium shapes of liquid lens. The solute is initialized in Red phase as the initial condition. The black solid line shows the contour of the concentration at [0, 0.1, 0.2, 0.3 , $0.4,0.5,0.6,0.7,0.8,0.9,1]$ respectively and the upper inset in the first picture is the stabilized condition of bulk phase (The black, dark gray, and light gray represent Blue phase, Red phase, and Green phase, respectively). The coordinates of $x, y$ axes are repreneted by the number of lattices. The time step (t.s. in short) is (a) 100 t.s., (b) 500 t.s., (c) 1000 t.s., (d) 10000 t.s., (e) 50000 t.s., (f) 100000 t.s. 


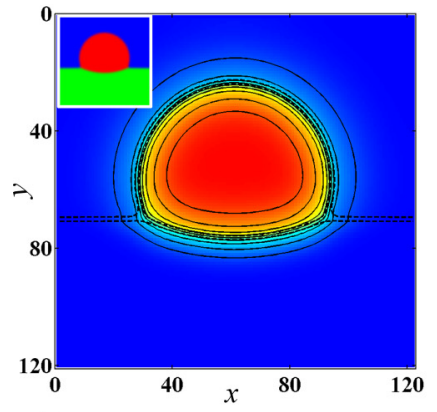

(a)

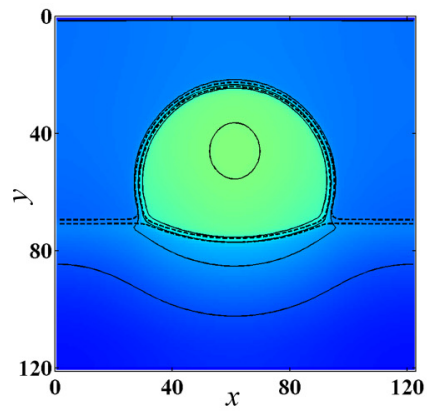

(d)

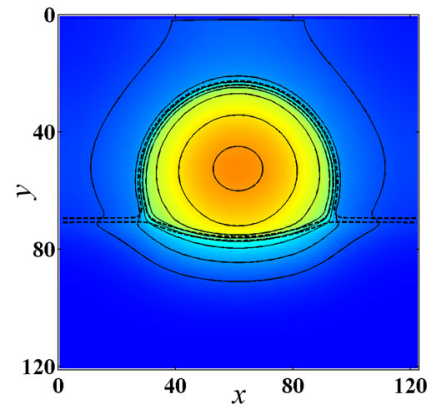

(b)

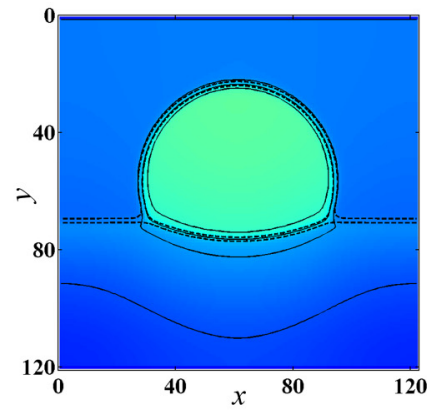

(e)

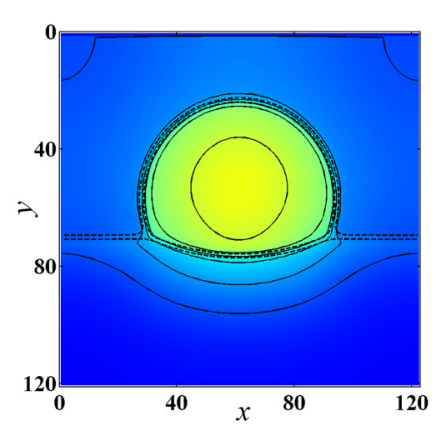

(c)

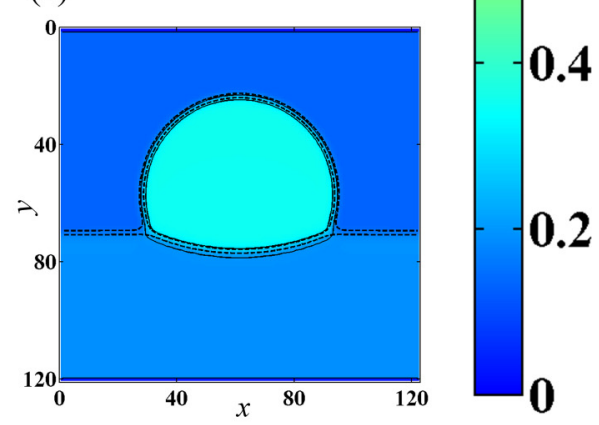

(f)

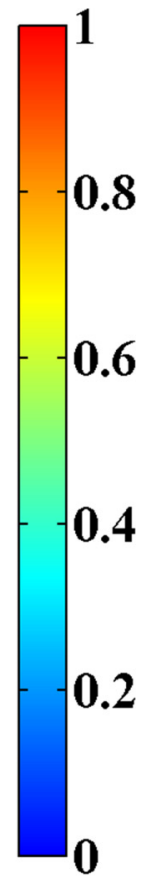

0.6

\section{2}

FIG. 9. The pseudocolor images representing the mass transfer of the three-phase soluble compound in the equilibrium shapes of the lens. The solute is initialized in Red phase. The black solid line shows the contour of the concentration at $[0,0.1,0.2,0.3,0.4,0.5,0.6,0.7,0.8,0.9$, 1], respectively, and the upper inset in the first picture is the stabilized condition of bulk phase (The black, dark gray, and light gray represent Blue phase, Red phase, and Green phase, respectively). The coordinates of $x, y$ axes are repreneted by the number of lattices. The time step (t.s. in short) is (a) 100 t.s., (b) 500 t.s., (c) 1000 t.s., (d)10000 t.s., (e) 50000 t.s., (f) 100000 t.s.

The resistance of the mass transfer is the most important characteristic of transport-limited phenomena in multiphase systems. It is usually limited by the refresh rate of the molecules arriving at the interface, represented by the diffusion parameter ratios. It shows that the resistance to mass transfer locates in the Green phase at the beginning, and an obvious concentration gradient is inside the liquid lens. That is because the diffusion parameter in Red phase is larger than that in Green phase and the mass transfer driving force caused by concentration gradient exists. After nearly 20,000 t.s., the diffusion becomes slow since the concentration in each phase gradually approaches the thermodynamic concentration. The final equilibrium state is reached after nearly 200,000 t.s. In addition, the equilibrium partial concentration in each phase is equal to the partition coefficient from Eq. (43).

Then, the three-phase soluble compound is testified in this case with the triple fluid junction. The parameter of bulk phase, the action of $\lambda_{k, k l}$ and the initialized condition are the same as before. In order to check the stability of the diffusion parameters, we choose the relaxation time $\tau_{d, B}=$ $0.7, \tau_{d, R}=0.6, \tau_{d, G}=0.55$, from which we get the diffusivity ratio $D_{d, B}: D_{d, R}: D_{d, G}=4: 2: 1$. In addition, we use the bounce back scheme for the boundary condition of the solute. Figure 9 gives the pseudocolor images of the concentration evolution of the concentrations at different instances of time. At the beginning, the action of mass transfer is also determined by the diffusion under the presence of the large driving force. The solute diffuses rapidly in Blue phase while more slowly in Green phase since the diffusion parameter in the Blue phase is larger. The concentration gradient in Red phase gradually decreases and the equilibrium of interface B-G is first reached. After that, it takes a long time to reach equilibrium state since the solute diffuses in the Green phase. In addition, when simulating with a wide range of relaxation time, it is found that the concentration at the interface fluctuates with a sharp change under the circumstance where the smallest relaxation time is less than 0.55. Despite of the limit of the smallest setting of the relaxation time, the largest diffusion parameter ratio could reach 20 with numerical stabilities.

\section{E. Modeling liquid-liquid mass transfer and reaction in a Janus droplet in microchannel}

The Janus droplet is one of the special types of microdroplets that two opposite droplets with different chemical properties adhere to each other [74,75]. Microfluidic technology provides a controllable and high throughput method to produce uniform micro-Janus droplets [76] and the hydrodynamic of the Janus droplet in microchannel has been studied [77-79]. The multiphase mass transfer and the interfacial reactions are other fundamental aspects for further understanding and applications of the Janus droplet when considering it as a microdroplet based reactor [80,81]. However, to our best knowledge, there are still few studies for monitoring the mixing performance or reactions in the multiphase droplet flow under the limit of the experiment set up. Specifically, the elaborate structure of the Janus droplet is another challenge to get in situ detection of the concentration 


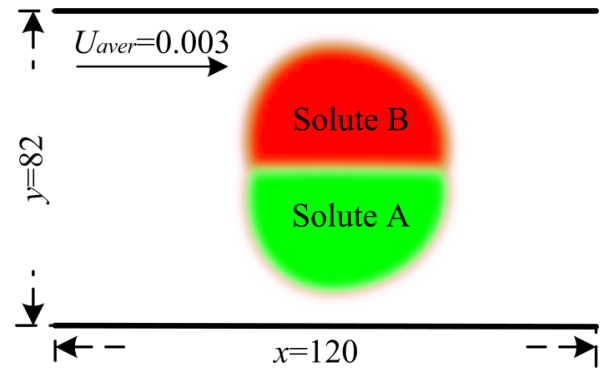

FIG. 10. Schematic of the initial condition of the dispersed phase Red (dark gray) and Green (light gray), the reactive dilute species A and B.

field in these microdevices. The mass transfer and reaction in microchannel could be classified into two steps [82,83]: the droplet formation process and the droplet transport process. The droplet transport process plays an important role in multiphase mass transfer condition that the percentage of mass transfer in the total steps could reach up to nearly $70 \%$ [84] and the mass-transfer could be easily adjusted through geometry construction or other active ways. The technology to achieve the real-time measurement of concentration has been developed through the $\mathrm{pH}$ indicators [85] or the micro-LIF (laser-induced fluorescence) technology [86]. In addition, the mass-transfer performance is measured by the overall masstransfer coefficient $[14,87]$. However, the mass-transfer for a Janus droplet flow is difficult as the measurement of overall mass-transfer coefficient would be failed. On one hand, some reaction process is determined by the mass transfer rate, where the concentration distribution of the solute is most important in this case. On the other hand, the real-time measurement is limited to the solutes system and the real mass transfer could be hard to achieve.

In this section we employ this newly established model to understand the mass transfer and reaction between two parts of the Janus droplet during the transport process in microchannels. The Marangoni effect is not taken into account since the mass transfer of dilute solute at interface does not lead to an interface fluctuation in microfluids. It is clear to demonstrate the understanding of interface renewal phenomenon as shown in Sec. III D. A $N_{x} \times N_{y}=120 \times 82$ computational domain is implemented with the periodic boundary along $x$ direction, and the solid wall at the upper and below adopt the half-way bounce back scheme as shown in Fig. 10. Since we investigate the mass transfer and the reaction between the two semi-parts of Janus droplet, the node along $x$ direction is set as 120 in order to reduce computational load. The parameters for the bulk phase are fixed as Table VI. The density keeps constant in the numerical simulation since effect of density ratio could be neglected in the liquid-liquid-liquid flow system of Janus formation or transport in microchannel [77]. Before adding the solutes into the system, we take 10000 steps for initializing the bulk phase where the positions of Red phase and Green phase follow Eqs. (51) and (52), respectively:

$$
\begin{aligned}
& \left(x-\frac{N_{x}}{2}\right)^{2}+\left(y-\frac{N_{y}}{2}\right)^{2} \leqslant R^{2} \text { with } y \leqslant \frac{N_{y}}{2}, \\
& \left(x-\frac{N_{x}}{2}\right)^{2}+\left(y-\frac{N_{y}}{2}\right)^{2} \leqslant R^{2} \text { with } y>\frac{N_{y}}{2},
\end{aligned}
$$

TABLE VI. Simulation parameters for mass transfer and reaction of Janus droplet transport in microchannel.

\begin{tabular}{llll}
\hline \hline Bulk phase & \multicolumn{1}{c}{ Phase B } & \multicolumn{1}{c}{ Phase R } & \multicolumn{1}{c}{ Phase G } \\
\hline Density & $\rho_{B}=1$ & $\rho_{R}=1$ & $\rho_{G}=1$ \\
Kinematic viscosity & $\mu_{B}=1 / 6$ & $\mu_{R}=1 / 6$ & $\mu_{G}=1 / 6$ \\
Interface tension & $\sigma_{B R}=0.01$ & $\sigma_{B G}=0.01$ & $\sigma_{R G}=0.005$ \\
Solute & Solute A & Solute B & Solute C \\
Standard concentration & $C_{A}^{0}=1$ & $C_{B}^{0}=1$ & $C_{C}^{0}=0$ \\
$C_{s}(t=0)$ & $C_{A}=C_{A}^{0} x_{G}$ & $C_{B}=C_{B}^{0} x_{B}$ & $C_{C}=0$ \\
$D_{s, B}$ & $1 / 60$ & $1 / 60$ & $1 / 60$ \\
$D_{s, R}$ & $1 / 60$ & $1 / 60$ & $1 / 60$ \\
$D_{s, G}$ & $1 / 30$ & $1 / 30$ & $1 / 30$ \\
$\lambda_{s, k l}$ & $\lambda_{s, k l}=1$ & $\lambda_{s, B}=1$ & $\lambda_{s, B G}=1.609$ \\
& & $\lambda_{s, k l}=1$ \\
\hline \hline
\end{tabular}

where $R$ is the radius of circle with 30 l.u. The rest is full of the Blue phase taken as the continuous phase, and the wetting properties are set as full of the Blue phase [50]. An average velocity $U_{\text {average }}=0.003$ is along the $x$ direction. The convergence shape of the Janus droplet is drawn in Fig. 10. Here we consider a first-order chemical reaction for solute A or B as the following way:

$$
A+B \stackrel{k_{r}}{\rightarrow} C \quad R_{s}=-k_{r} C_{A} C_{B}
$$

where $k_{r}$ is the chemical reaction rate constant. The reaction source term $R_{S}$ is added into the governing equation term in Eq. (25), forming the convection-diffusion-reaction governing equations with an interface topology:

$$
\begin{aligned}
& \partial_{t} C_{A}+\partial_{x \alpha} N_{A}-R_{s}=0, \\
& \partial_{t} C_{B}+\partial_{x \alpha} N_{B}-R_{s}=0, \\
& \partial_{t} C_{C}+\partial_{x \alpha} N_{C}+R_{s}=0 .
\end{aligned}
$$

The diffusivity in Green phase is twice of that in Red phase. The solute A has a partition coefficient compound in Red and Green phases, while the solute B and solute $\mathrm{C}$ are defined only in Red phase. The Henry coefficient for the solute $\mathrm{A}$ is $H_{R G}=C_{A, R} / C_{s, G}=5$ in this numerical case. The physical parameters are given in Table VI. The flow condition modified by two key dimensionless numbers in Janus transport is in agreement with Ref. [77], where the Capillary number $\mathrm{Ca}=$ $0.05=U_{\text {aver }} \mu_{B} / \sigma_{B R}$ and the Reynolds number $\operatorname{Re}=1.4=$ $\rho_{B} U_{\text {aver }} L / \mu_{B}$ with the channel width $L=80$ are calculated from the fixed parameter.

The numerical results are shown as pseudo-color images of the concentration profile of each solute at different instances of time in Fig. 11. The Peclet number is $\mathrm{Pe}=14.4=$ $U_{\text {aver }} L / D_{s, G}$, indicating a convection dominated condition. Especially, fluid recirculation inside the two parts of Janus droplet enhances the convection as the droplet transport in the straight channels and the recirculation vortex are located in each part of Red phase and Green phase at the top and bottom respectively. In order to represent the mass transfer and reaction process inside the droplet, it is important to know the feature of the transport-time reaction system. The characteristic reaction time of the first reaction system of solute A can be described as $t_{r}=\left(k_{r} C_{A}^{0}\right)^{-1}$. The characteristic 


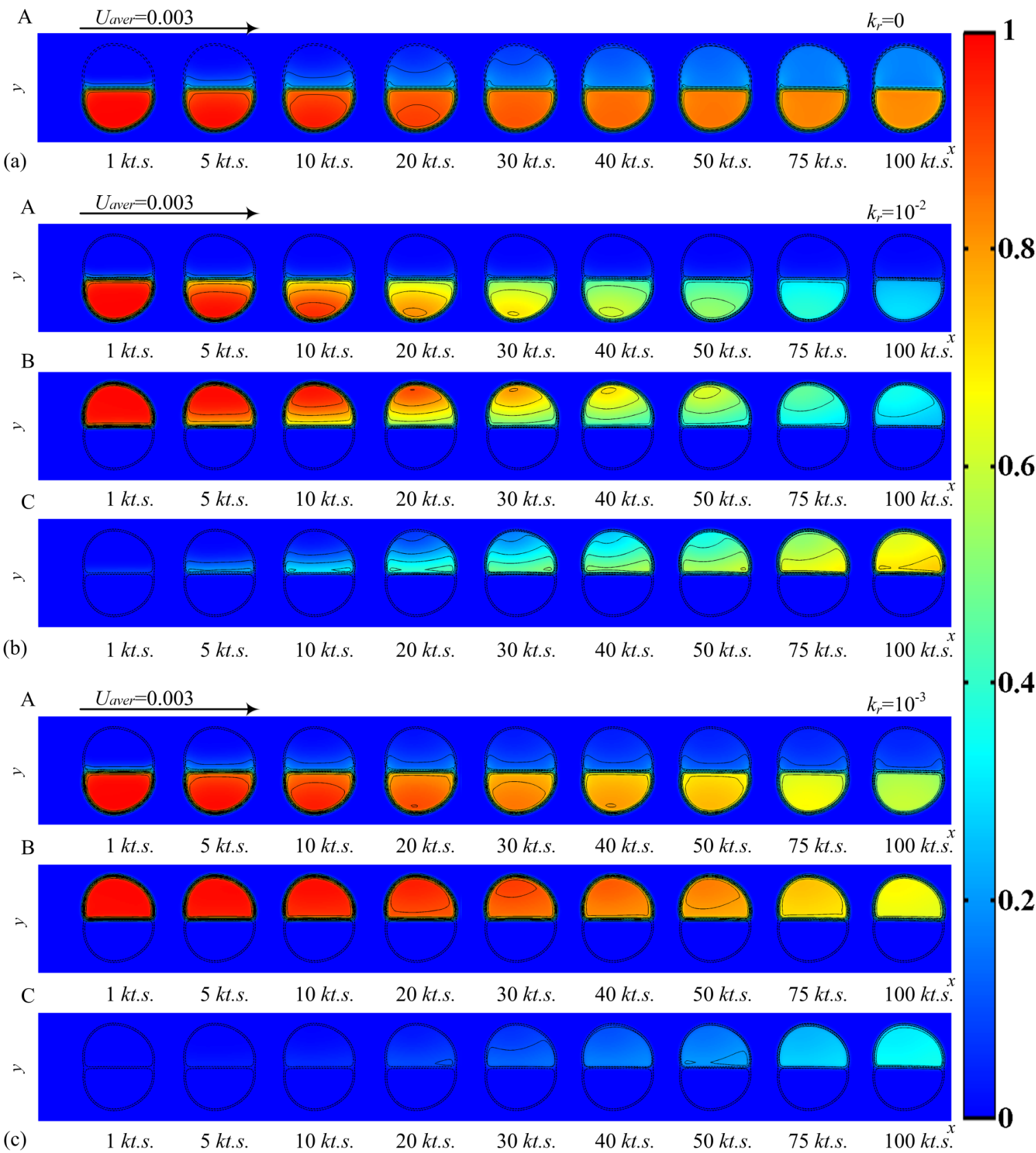

FIG. 11. Pseudocolor images of concentration profile of each solute compound at instant times during the mass transfer and reaction in the Janus droplet flowing through microchannels. An average velocity $U_{\text {aver }}=0.0031 . u$. $/$ t.s. is toward the right side of $x$ direction. The black solid line shows the contour of the concentration at $[0,0.1,0.2,0.3,0.4,0.5,0.6,0.7,0.8,0.9,1]$, respectively. The time step (kt.s. in short) is shown in the gragh. The reaction rate parameter (a) $k_{r}=0$, (b) $k_{r}=10^{-2}$, (c) $k_{r}=10^{-3}$.

diffusivity time is $t_{d}=L^{2} / 2 D_{s, G}$ [88]. In Sec. III C our model is compared with Higbie's penetration theory, where the surface renewal rate is related to the diffusivity time so that we can compare the ratio $t_{d} / t_{r}=k_{r} L^{2} / 2 D_{s, G}$ of the characteristic diffusivity time to characteristic reaction time with the reaction and diffusion rate through the interface. In this numerical simulation, the solute A penetrates the interface R-G into Red phase and the reaction occurs when the solute A reaches the solute B. Moreover, in order to make a comparison, the mass transfer of solute A without reaction term shown in Fig. 11(a). Take the case $k_{r}=10^{-2}$ with $t_{d} / t_{r}=1920$ for example, Fig. 11(b) demonstrates the concentration profile of solutes at instants of the transport times. The reaction occurs immediately as the solute A crosses the interfaces. An obvious concentration gradient of solutes A exists in the Green phase and the largest concentration of solute $A$ at each time is presented in the bottom of the Green part since the reaction happens in the other part of Janus droplet. This result is also consistent with the behavior of solute B simultaneously. The concentration profile of solute $\mathrm{C}$ results from the chemical reaction at the interface between two parts of Janus droplet and transfers into the whole part from the interface. The process is 
dominated by diffusivity since the reaction is a fast process compared with the diffusion. On the contrary, Fig. 11(c) shows the reaction-dependent case as $t_{d} / t_{r}=192$. Since the characteristic time of reaction is apparently larger than that of diffusion, apparent concentration profile of solute A in Red phase exists due to the interface renewal. The distribution of the reactants and the reaction rate lead to the moderate reactions in Red phase. Therefore, the product of solute $\mathrm{C}$ generates not only at the interface but also in the area where the Solute A can reach. Inconspicuous transport concentration gradient is observed from Fig. 11(c). These results could be explained by diffusion-dominant or reaction-dominant phenomenon. The diffusion compared with the reaction is a slow process, which becomes the resistance for reaction to happen. Therefore, a sharp gradient of concentration happens in the limit of transport of the solute compound. In the opposite, the diffusion is the key to dominate the reaction process, making a mild concentration distribution in the Janus transport system. In addition, the mass transfer of solute $A$ to Red phase is enhanced by the chemical reaction as shown in the comparison of Fig. 11. The consumption of solute A leads to intensification of the mass transfer driving force, which reduces the resistance at the interface film. Usually, the mass transfer enhancement by reaction is represented by the enhancement factor $E$ [89] in overall measurement of the process, based on the stagnant film theory. However, this treatment fails in the sophisticated case without giving the qualitative details of the concentration profile. The enhancement for the multiphase transfer in the Janus droplet is well presented not only for the degree of transport enhancement but also for the distribution profiles in the multiphase with numerical stability. In addition, simulations with this technique would be able to explore the mixing performance with mass transfer and reaction in the aspect of three phases and brings a new insight to interphase transport of solute compound $[90,91]$.

\section{SUMMARY}

In this paper, a LB-based framework for modeling the interphase mass transfer of dilute solute in a three-phase system is proposed. A new collision operator Eq. (30) for capturing the solute interface behaviors is incorporated into three-phase systems. The Chapman-Enskog analysis demonstrates that the Maxwell-Stefan equation is recovered and the equation of state is demonstrated. In addition, by comparing the analysis with the thermodynamics of the solute distribution, this algorithm is well posed and is able to simulate the solute distribution in three-phase systems, including compound soluble in one phase, compound adsorbed on single-interface, compound in two phases, and solute soluble in three phases. In order to verify the presented model, several numerical cases such as phase decomposition, multilayered planar interfaces, and liquid lens have been performed. It is found that the thermodynamic equilibrium with partition coefficient is consistent with the theoretical analysis. Moreover, the multiphase mass transfer is in good agreement with the penetration theory and this problem is also examined in a triple fluid junction system. The presented model is capable and reliable for studying the multiphase transfer of dilute compound in a three-phase flow.
Furthermore, we applied the new model to the multiphase mass transfer and reaction of Janus droplet transport in a straight microchannel. The dynamic mass transfer and reaction of dilute solute with a partition coefficient is well captured. Modeling the multiphase mass transfer is an attractive problem, especially for the microdroplet dynamics in the microfluidics. The presented model is established for a threephase system in terms of efficiency and numerical stability. This construction can be easily implemented for mass transfer problems in three-phase flows. The methodology would be applied in our future works, such as mixing performance of double or multiemulsion during the formation process in microchannel and solute mass transfer and reaction of multiphase flow with partition coefficients among the threephase flows.

\section{ACKNOWLEDGMENTS}

Financial support from National 973 Project of PR China (Grant No. 2013CB733604), National Natural Science Foundation (Grant No. 21576151), and Tsinghua Fudaoyuan Research Fund are acknowledged.

\section{APPENDIX: CHAPMAN-ENSKOG ANALYSIS OF THE PRESENT LB MODEL FOR CONVECTION-DIFFUSION-REACTION IN MULTIPHASE SYSTEMS}

Here we present the Chapman-Enskog analysis to demonstrate the consistency and the error of the advection-diffusionreaction model with interface topology as proposed in Eq. (31). For the lattice consistency, the useful properties are easily given as the LBM scheme as

$$
\begin{aligned}
& \sum_{i}^{q} w_{i}=1, \quad \sum_{i}^{q} w_{i} e_{i \alpha}=0, \\
& \sum_{i}^{q} w_{i} e_{i \alpha} e_{i \beta}=c_{s}^{2} \delta_{\alpha \beta}, \quad \sum_{i}^{q} \frac{w_{i} e_{i \alpha} e_{i \beta}}{\left\|e_{i \alpha}\right\|}=2 k \delta_{\alpha \beta}, \\
& \sum_{i}^{q} w_{i} e_{i \alpha} e_{i \beta} e_{i \gamma}=0,
\end{aligned}
$$

where $k$ is the geometric constant, which is 0.153 in D2Q9 and 0.134 in D3Q19, for example. According to Eq. (28), the equilibrium distribution function, the following movement can be derived based on the properties as above:

$$
\begin{aligned}
& \sum_{i}^{q} w_{i} d_{i, s}^{\mathrm{eq}}=C_{s}, \quad \sum_{i}^{q} e_{i \alpha} d_{i, s}^{\mathrm{eq}}=C_{s} U_{\alpha} \\
& \sum_{i}^{q} w_{i} e_{i \alpha} e_{i \beta} d_{i, s}^{\mathrm{eq}}=C_{s} c_{s}^{2} \delta_{\alpha \beta}+C_{s} U_{\alpha} U_{\beta}, \\
& \sum_{i}^{q} w_{i} e_{i \alpha} e_{i \beta} e_{i \gamma} e d_{i, s}^{\mathrm{eq}}=C_{s} c_{s}^{2}\left(U_{\alpha} \delta_{\beta \gamma}+U_{\beta} \delta_{\alpha \gamma}+U_{\gamma} \delta_{\alpha \beta}\right) .
\end{aligned}
$$

In this Appendix, the interface collision operator is taken as Eq. (A3) for simplicity, where only the collision operator 
of interface $k l$ is expanded and the other interface profiles have the same expression with different interface vector $F_{k l \alpha}$, interface function $W_{s}$, and the interface parameter $\beta_{s, k l}$ :

$$
\Omega_{s, 2}^{i}=\beta_{s, k l} W_{s} d_{i, s}^{(\mathrm{eq}, 0)} \frac{e_{i \alpha} F_{k l \alpha}}{\left\|e_{i \alpha}\right\|\left\|F_{k l \alpha}\right\|} .
$$

From Eq. (A3), one can derive the following moment conditions with Eq. (A1) properties:

$$
\begin{aligned}
& \sum_{i}^{q} \Omega_{s, 2}^{i}=0, \\
& \sum_{i}^{q} e_{i \alpha} \Omega_{s, 2}^{i}=2 k \beta_{s, k l} W_{s} C_{s} F_{\alpha} /\left\|F_{\alpha}\right\|, \\
& \sum_{i}^{q} e_{i \alpha} e_{i \beta} \Omega_{s, 2}^{i}=0 .
\end{aligned}
$$

Chapman-Enskog analysis uses two time scales to derive the macroscopic equations, and the time derivative is expanded at the convective time scale $\partial_{t}^{(1)}$ and the diffusion time scale $\partial_{t}^{(2)}$ in different order of $\varepsilon: \partial_{t}=\varepsilon \partial_{t}^{(1)}+\varepsilon^{2} \partial_{t}^{(2)}$. In addition, the spatial derivative is associated with $\partial_{x \alpha}=\varepsilon \partial_{x \alpha}^{(1)}$.
We use the Taylor series expansion of the left-hand side and the right-hand side of Eq. (25), respectively, around the local equilibrium distribution at fourth order,

$$
\begin{gathered}
d_{i, s}\left(x_{\alpha}+e_{i \alpha} \Delta t, t+\Delta t\right)=\sum_{n=0}^{\infty} \frac{\Delta t^{n}}{n !}\left(\partial_{t}+e_{i \alpha} \partial_{x \alpha}\right)^{n} d_{i, s}\left(x_{\alpha}, t\right), \\
d_{i, s}=d_{i, s}^{(0)}+\varepsilon d_{i, s}^{(1)}+\varepsilon^{2} d_{i, s}^{(2)}+\varepsilon^{3} d_{i, s}^{(3)}+O\left(\varepsilon^{4}\right), \\
\Omega_{2}^{i}=\Omega_{2}^{(0) i}+\varepsilon \Omega_{2}^{(1) i}+\varepsilon^{2} \Omega_{2}^{(2) i}+\varepsilon^{3} \Omega_{2}^{(3) i}+O\left(\varepsilon^{4}\right), \\
R_{s}=R_{s}^{(0)}+\varepsilon R_{s}^{(1)}+\varepsilon^{2} R_{s}^{(2)}+\varepsilon^{3} R_{s}^{(3)}+O\left(\varepsilon^{4}\right) .
\end{gathered}
$$

One can group the governing equations into a series of equations in terms of the same order in $\varepsilon$,

$$
\varepsilon^{0}: \frac{1}{\tau_{s}}\left(d_{i, s}^{\mathrm{eq}}-d_{i, s}^{(0)}\right)+\Omega_{2}^{(0) i}+\Delta t w_{i} R_{s}^{(0)}=0,
$$

where the definition of the successive approximations leads to $d_{i, s}^{\text {eq }}-d_{i, s}^{(0)}$, which implies that $\Omega_{2}^{(0) i}=0, R_{s}^{(0)}=0$. It also obeys the conservation of mass law. With the help of Eq. (A7), the higher order of $\varepsilon$ can be arranged as

$$
\begin{gathered}
\varepsilon^{1}: \frac{1}{\tau_{s}} d_{i, s}^{(1)}=-\Delta t\left(\partial_{t}^{(1)}+e_{i \alpha} \partial_{x \alpha}^{(1)}\right) d_{i, s}^{(0)}+\Omega_{2}^{(1) i}+\Delta t w_{i} R_{s}^{(1)}, \\
\varepsilon^{2}: \frac{1}{\tau_{s}} d_{i, s}^{(2)}=-\Delta t \partial_{t}^{(2)} d_{i, s}^{(0)}+\Delta t^{2}\left(\tau_{s}-\frac{1}{2}\right)\left(\partial_{t}^{(1)}+e_{i \alpha} \partial_{x \alpha}^{(1)}\right)^{2} d_{i, s}^{(0)}-\Delta t\left(\partial_{t}^{(1)}+e_{i \alpha} \partial_{x \alpha}^{(1)}\right) \tau_{s} \Omega_{2}^{(1) i}+\Omega_{2}^{(2) i}+\Delta t w_{i} R_{s}^{(2)}, \quad(\mathrm{A} 9) \\
\varepsilon^{3}: \frac{1}{\tau_{s}} d_{i, s}^{(3)}=-\Delta t \partial_{t}^{(3)} d_{i, s}^{(0)}+\Delta t^{2}\left(2 \tau_{s}-\frac{1}{2}\right)\left(\partial_{t}^{(1)}+e_{i \alpha} \partial_{x \alpha}^{(1)}\right) \partial_{t}^{(2)} d_{i, s}^{(0)}-\Delta t^{3}\left(\tau_{s}^{2}-\tau_{s}+\frac{1}{6}\right)\left(\partial_{t}^{(1)}+e_{i \alpha} \partial_{x \alpha}^{(1)}\right)^{3} d_{i, s}^{(0)}-\Delta t \tau_{s} \partial_{t}^{(2)} \Omega_{2}^{(1) i} \\
+\Delta t^{2} \tau_{s}\left(\tau_{s}-\frac{1}{2}\right)\left(\partial_{t}^{(1)}+e_{i \alpha} \partial_{x \alpha}^{(1)}\right)^{2} \Omega_{2}^{(1) i}-\Delta t \tau_{s}\left(\partial_{t}^{(1)}+e_{i \alpha} \partial_{x \alpha}^{(1)}\right) \Omega_{2}^{(2) i} \\
+\Omega_{2}^{(3) i}-\Delta t^{2} \tau_{s}\left(\partial_{t}^{(1)}+e_{i \alpha} \partial_{x \alpha}^{(1)}\right) w_{i} R_{s}^{(2)}+\Delta t w_{i} R_{s}^{(3)} .
\end{gathered}
$$

The local distribution function imposes the following conservation of mass based on the isotropy of the lattice tensors in Eq. (A6), and it assumes that the higher order of the equilibrium distribution makes no contribution to the local mass,

$$
\begin{aligned}
& \sum_{i}^{q} d_{i, s}^{(0)}=0 \sum_{i}^{q} e_{i \alpha} d_{i, s}^{(0)}=0 \sum_{i}^{q} e_{i \alpha} e_{i \beta} d_{i, s}^{(0)}=\rho_{s} c_{s}^{2} \delta_{\alpha \beta}, \\
& \sum_{i}^{q} d_{i, s}^{(n)}=0 \quad \text { for } n>0 .
\end{aligned}
$$

By summing $\sum_{i}$ Eq. (A8) with the help of Eqs. (A11) and (A12), and taking $\sum_{i}$ Eq. (A9) with the help of Eqs. (A11)-(A13), and then summing $\sum_{i}$ Eq. (A10) with the help of Eqs. (A11)-(A14), one can obtain the governing equations in terms of three time scales, respectively,

$$
\begin{gathered}
\varepsilon^{1}: \partial_{t}^{(1)} C_{s}+\partial_{x \alpha}^{(1)} C_{s} U_{\alpha}=0 \\
\varepsilon^{2}: \partial_{t}^{(2)} C_{s}=\Delta t c_{s}^{2}\left(\tau_{s}-\frac{1}{2}\right) \partial_{x \alpha}^{(1)} \partial_{x \alpha}^{(1)} C_{s}-\tau_{s} \partial_{x \alpha}^{(1)} \sum_{i=1}^{q} e_{i \alpha} \Omega_{2}^{(1) i}+\frac{1}{\Delta t} \Omega_{2}^{(2) i}+R_{s}^{(2)}+\partial_{x \alpha}^{(1)}\left[C_{s}\left(\partial_{t}^{(1)} U_{\alpha}+U_{\beta} \partial_{x \beta}^{(1)} U_{\alpha}\right)\right]
\end{gathered}
$$




$$
\begin{aligned}
\varepsilon^{3}: \partial_{t}^{(3)} C_{s}= & -\Delta t^{2}\left(\tau_{s}^{2}-\tau_{s}+\frac{1}{6}\right)\left\{2 \partial_{t}^{(1)} \partial_{x \alpha}^{(1)}\left[C_{s}\left(\partial_{t}^{(1)} U_{\alpha}+U_{\beta} \partial_{x \beta}^{(1)} U_{\alpha}\right)\right]+3 c_{s}^{2} \partial_{t}^{(1)} \partial_{x \alpha}^{(1)} \partial_{x \alpha}^{(1)} C_{s}\right. \\
& \left.+\partial_{x \alpha}^{(1)} \partial_{x \beta}^{(1)}\left(\partial_{t}^{(1)} C_{s} U_{\alpha} U_{\beta}+c_{s}^{2} \partial_{x \gamma}^{(1)}\left[C_{s}\left(U_{\alpha} \delta_{\beta \gamma}+U_{\beta} \delta_{\alpha \gamma}+U_{\gamma} \delta_{\alpha \beta}\right)\right]\right)\right\} \\
& +\Delta t \tau_{s}\left(2 \tau_{s}-1\right)\left(\partial_{t}^{(1)} \partial_{x \alpha}^{(1)} \sum_{i=1}^{q} e_{i \alpha} \Omega_{2}^{(1) i}\right)-\tau_{s}\left(\partial_{x \alpha}^{(1)} \sum_{i=1}^{q} e_{i \alpha} \Omega_{2}^{(2) i}\right)-\Delta t \tau_{s} \partial_{t}^{(1)} R_{s}^{(2)}+R_{s}^{(3)} .
\end{aligned}
$$

Combining Eq. (A13) at $\varepsilon^{1}$ scales, Eq. (A14) at $\varepsilon^{2}$ scales, and Eq. (A15) at $\varepsilon^{3}$ scales, we have

$$
\partial_{t} C_{s}+\partial_{x \alpha} C_{s} U_{\alpha}=\Delta t c_{s}^{2}\left(\overline{\tau_{s}}-\frac{1}{2}\right) \partial_{x \alpha}^{2} C_{s}-\tau_{s} \partial_{x \alpha} \sum_{i=1}^{q} e_{i \alpha} \Omega_{d, 2}^{i}+R_{s}+\text { Error, }
$$

where Error is the error part at $O\left(\varepsilon^{4}\right)$, and this governing equation can be rewritten as the convection-diffusion-reaction equation with an interface tropology with the property of the interface collision as Eq. (A4),

$$
\partial_{t} C_{s}+\partial_{x \alpha}\left[C_{s} U_{\alpha}-D_{s} \partial_{x \alpha} C_{s}+2 k C_{s} \tau_{s} \beta_{s} W_{s}\left(x_{k}\right) F_{k l \alpha} /\left\|F_{k l \alpha}\right\|\right]-R_{s}=\text { Error, }
$$

in which the diffusion coefficient equals to $D_{s}=\Delta t c_{s}^{2}\left(\overline{\tau_{s}}-0.5\right)$. Therefore, we can clearly see that the concentration flux can be clearly recovered from the LB with the expression

$$
N_{\alpha}=C_{s} U_{\alpha}-D_{s} \partial_{x \alpha} C_{s}+2 k C_{s} \tau_{s} \beta_{s} W_{s}\left(x_{k}\right) F_{k l \alpha} /\left\|F_{k l \alpha}\right\| .
$$

In the above equation, the interface collision operator is related to a macro equation as $2 k C_{s} \tau_{s} \beta_{s} W_{s}\left(x_{k}\right) F_{k l \alpha} /\left\|F_{k l \alpha}\right\|$. Without the interplay of this part, Eq. (A17) is degenerated into the convection-diffusion-reaction equation as proposed by Ref. $[68,69,92]$.

Note that the interface operator is local and linear, so much concern should be paid into the Error part derived from the analytical relations. Here we represent the Error term with an order of $O\left(\varepsilon^{4}\right)$ to analyze the novel term we proposed to the interface profile and the reaction term,

$$
\begin{aligned}
\text { Error }= & \left(1-\Delta t^{2}\left(2 \tau_{s}-1\right)\left(\tau_{s}-\frac{1}{3}\right) \partial_{t}\right) \partial_{x \alpha}\left[C_{s}\left(\partial_{t} U_{\alpha}+U_{\beta} \partial_{x \beta} U_{\alpha}\right)\right] \\
& -\Delta t D_{s}\left(\tau_{s}-\frac{1}{3}\right)\left[3 \partial_{t} \partial_{x \alpha}^{2} C_{s}+\frac{1}{c_{s}^{2}} \partial_{x \alpha} \partial_{x \beta}\left(\partial_{t} C_{s} U_{\alpha} U_{\beta}+c_{s}^{2} \partial_{x \gamma}\left[C_{s}\left(U_{\alpha} \delta_{\beta \gamma}+U_{\beta} \delta_{\alpha \gamma}+U_{\gamma} \delta_{\alpha \beta}\right)\right]\right)\right] \\
& +\Delta t 2\left(2 \tau_{s}-1\right) k C_{s} \tau_{s} \beta_{s} W_{s}\left(\partial_{t} \partial_{x \alpha} \frac{F_{k l \alpha}}{\left\|F_{k l \alpha}\right\|}\right)-\Delta t \tau_{s} \partial_{t} R_{s}+O\left(\varepsilon^{4}\right) .
\end{aligned}
$$

This error term can be removed or reduced under certain conditions. Under the limit of $O(M a)$ and the low Reynolds number, the first and second terms can be neglected. We propose a partition parameter $\lambda_{s}$ to derive the parameter $\beta_{s}$ linearly, implying that the first-linear operator may fail to predict the interface profile at a higher value of $\lambda_{s}$. We prove that this partition coefficient is up to 100 without the influence on the error part. In addition, the reaction term needs to be $R_{s} \ll 1$ to reach the accuracy.

[1] X. Casadevall I Solvas and A. DeMello, Chem. Commun. 47, 1936 (2011).

[2] L. Shui, J. C. T. Eijkel, and A. van den Berg, Adv. Colloid Interfac. 133, 35 (2007).

[3] C. Zhao, Adv. Drug Deliver. Rev. 65, 1420 (2013).

[4] A. Fernández-Nieves, V. Vitelli, A. S. Utada, D. R. Link, M. Márquez, D. R. Nelson, and D. A. Weitz, Phys. Rev. Lett. 99, 157801 (2007).

[5] L. Zhang, S. Hao, B. Liu, H. C. Shum, J. Li, and H. Chen, ACS Appl. Mater. Inter. 5, 11489 (2013).

[6] H. Zhao, T. Chan, B. Merriman, and S. Osher, J. Comput. Phys. 127, 179 (1996).

[7] K. A. Smith, F. J. Solis, and D. Chopp, Interface. Free Bound. 4, 263 (2002).

[8] R. I. Saye and J. A. Sethian, Proc. Natl. Acad. Sci. USA 108, 19498 (2011).
[9] R. Bonhomme, J. Magnaudet, F. Duval, and B. Piar, J. Fluid Mech. 707, 405 (2012).

[10] H. G. Lee and J. Kim, Physica A 423, 33 (2015).

[11] F. Boyer and C. Lapuerta, ESAIM: Math. Modell. Numer. Anal. 40, 653 (2006).

[12] J. Kim, Comput. Method. Appl. M. 198, 3105 (2009).

[13] N. Raimondi and L. Prat, AIChE J. 57, 1719 (2011).

[14] N. Di Miceli Raimondi, L. Prat, C. Gourdon, and P. Cognet, Chem. Eng. Sci. 63, 5522 (2008).

[15] T. Dietrich, Microchemical Engineering in Practice (John Wiley \& Sons, New York, 2011).

[16] D. Bothe, Calculations and simulations, Microchem. Eng. Pract., doi:10.1002/9780470431870.ch7.

[17] M. R. Davidson and M. Rudman, Numer. Heat Transfer, Part B 41, 291 (2002).

[18] M. Ohta and M. Suzuki, Solvent Extr. Res. Dev. 3, 138 (1996). 
[19] J. Petera and L. R. Weatherley, Chem. Eng. Sci. 56, 4929 (2001).

[20] C. Yang and Z. Mao, Chem. Eng. Sci. 60, 2643 (2005).

[21] A. Onea, M. Woerner, and D. G. Cacuci, Chem. Eng. Sci. 64, 1416 (2009).

[22] Q. Li, K. H. Luo, Q. J. Kang, Y. L. He, Q. Chen, and Q. Liu, Prog. Energ. Combust. 52, 62 (2016).

[23] D. Lycett-Brown and K. H. Luo, Phys. Rev. E 94053313 (2016).

[24] C. K. Aidum and J. R. Clausen, Annu. Rev. Fluid Mech. 42, 439 (2010).

[25] A. K. Gunstensen, D. H. Rothman, S. Zaleski and G. Zanetti, Phys. Rev. A 43, 4320 (1991).

[26] X. Shan and H. Chen, Phys. Rev. E 47, 1815 (1993).

[27] M. R. Swift, E. Orlandini, W. R. Osborn, and J. M. Yeomans, Phys. Rev. E 54, 5041 (1996).

[28] X. He, X. Shan, and G. D. Doolen, Phys. Rev. E 57, R13 (1998).

[29] M. M. Dupin, I. Halliday, and C. M. Care, J. Phys. A: Gen. Phys. 36, 8517 (2003).

[30] T. J. Spencer, I. Halliday, and C. M. Care, Phys. Rev. E 82, 066701 (2010).

[31] S. Leclaire, M. Reggio, and J. Trépanier, J. Comput. Phys. 246, 318 (2013).

[32] X. Shan and H. Chen, Phys. Rev. E 49, 2941 (1994).

[33] Y. Shi, G. H. Tang, and Y. Wang, J. Comput. Phys. 314, 228 (2016).

[34] H. Chen, B. M. Boghosian, P. V. Coveney, and M. Nekovee, P. Roy. Soc. A-Math. Phy. 456, 2043 (2000)

[35] C. Semprebon, T. Kruger, and H. Kusumaatmaja, Phys. Rev. E 93, 033305 (2016).

[36] H. Liang, B. C. Shi, and Z. H. Chai, Phys. Rev. E 93, 013308 (2016).

[37] H. Liu, Q. Kang, C. R. Leonardi, S. Schmieschek, A. Narváez, B. D. Jones, J. R. Williams, A. J. Valocchi, and J. Harting, Computat. Geosci. 20, 777 (2016).

[38] X. Shan and G. Doolen, Phys. Rev. E 54, 3614 (1996).

[39] X. He and G. D. Doolen, J. Stat. Phys. 107, 309 (2002).

[40] L. S. Luo and S. S. Girimaji, Phys. Rev. E 67, 036302 (2003).

[41] M. E. McCracken and J. Abraham, Phys. Rev. E 71, 046704 (2005).

[42] N. I. Prasianakis and I. V. Karlin, Phys. Rev. E 78, 016704 (2008).

[43] N. I. Prasianakis and I. V. Karlin, Phys. Rev. E 76, 016702 (2007).

[44] E. Alper, Mass Transfer with Chemical Reaction in Multiphase Systems: Volume I: Two-Phase Systems. Volume II: Three-Phase Systems (Springer Science \& Business Media, Berlin, 2013), Vol. 72.

[45] I. Ginzburg, Adv. Water Resour. 28, 1171 (2005).

[46] D. Bin, S. Bao-Chang, and W. Guang-Chao, Chinese Phys. Lett. 22, 267 (2005).

[47] B. Shi, B. Deng, R. Du, and X. Chen, Comput. Math. Appl. 55, 1568 (2008).

[48] Z. Chai and T. S. Zhao, Phys. Rev. E 87, 063309 (2013).

[49] A. Riaud, S. Zhao, K. Wang, Y. Cheng, and G. Luo, Phys. Rev. E 89, 053308 (2014).

[50] Y. Fu, S. Zhao, L. Bai, Y. Jin, and Y. Cheng, Chem. Eng. Sci. 146, 126 (2016).

[51] H. Liu, A. J. Valocchi, and Q. Kang, Phys. Rev. E 85, 046309 (2012).
[52] W. Wang, S. Zhao, T. Shao, M. Zhang, Y. Jin, and Y. Cheng, Chem. Eng. Sci. 84, 148 (2012).

[53] P. L. Bhatnagar, E. P. Gross, and M. Krook, Phys. Rev. 94, 511 (1954).

[54] T. Reis and T. N. Phillips, J. Phys. A-Math. Theor. 40, 4033 (2007).

[55] S. V. Lishchuk, C. M. Care, and I. Halliday, Phys. Rev. E 67, 036701 (2003).

[56] X. Y. He, Q. S. Zou, L. S. Luo, and M. Dembo, J. Stat. Phys. 87, 115 (1997).

[57] A. Ladd and R. Verberg, J. Stat. Phys. 104, 1191 (2001).

[58] Z. Guo, C. Zheng, and B. Shi, Phys. Rev. E 65, 046308 (2002).

[59] D. Lycett-Brown and K. H. Luo, Phys. Rev. E 91, 023305 (2015).

[60] Q. Li, K. H. Luo, and X. J. Li, Phys. Rev. E 86, 016709 (2012).

[61] P. J. A. M. Kerkhof and M. A. M. Geboers, Chem. Eng. Sci. 60, 3129 (2005)

[62] R. Krishna and J. A. Wesselingh, Chem. Eng. Sci. 52, 861 (1997).

[63] R. B. Bird and D. J. Klingenberg, Adv. Water Resour. 62, 238 (2013).

[64] J. Zudrop, S. Roller, and P. Asinari, Phys. Rev. E 89, 053310 (2014).

[65] X. Shan, Phys. Rev. E 81, 045701 (2010).

[66] J. W. Cahn and J. E. Hilliard, J. Chem. Phys. 28, 258 (1958).

[67] J. W. Cahn and J. E. Hilliard, J. Chem. Phys. 31, 688 (1959).

[68] T. Inamuro, M. Yoshino, H. Inoue, R. Mizuno, and F. Ogino, J. Comput. Phys. 179, 201 (2002).

[69] Y. Huidan, L. Li-Shi, and S. S. Girimaji, Int. J. Comput. Eng. Sci. 3, 73 (2002).

[70] W. K. Lewis and W. G. Whitman, Ind. Eng. Chem 16, 1215 (1924).

[71] R. Higbie, The Rate of Absorption of a Pure Gas into Still Liquid During Short Periods of Exposure (1935).

[72] P. V. Danckwerts, Ind. Eng. Chem. 43, 1460 (1951).

[73] M. Latva-Kokko and D. H. Rothman, Phys. Rev. E 71, 056702 (2005).

[74] G. T. Vladisavljević, Particuology 24, 1 (2016).

[75] S. Yang, S. Kim, J. Lim, and G. Yi, J. Mater. Chem. 18, 2177 (2008).

[76] S. Lone and I. W. Cheong, RSC Adv. 4, 13322 (2014).

[77] T. Nisisako and T. Torii, Adv. Mater. 19, 1489 (2007).

[78] K. P. Yuet, D. K. Hwang, R. Haghgooie, and P. S. Doyle, Langmuir 26, 4281 (2010).

[79] C. Chen, R. K. Shah, A. R. Abate, and D. A. Weitz, Langmuir 25, 4320 (2009).

[80] R. Seemann, M. Brinkmann, T. Pfohl, and S. Herminghaus, Rep. Prog. Phys. 75, 016601 (2012).

[81] H. Song, D. L. Chen, and R. F. Ismagilov, Angew. Chem. Int. Ed. 45, 7336 (2006).

[82] Y. Zhao, G. Chen, and Q. Yuan, AIChE J. 53, 3042 (2007).

[83] J. D. Tice, H. Song, A. D. Lyon, and R. F. Ismagilov, Langmuir 19, 9127 (2003).

[84] J. Tan, Y. C. Lu, J. H. Xu, and G. S. Luo, Chem. Eng. J. 185, 314 (2012).

[85] J. H. Xu, J. Tan, S. W. Li, and G. S. Luo, Chem. Eng. J. 141, 242 (2008).

[86] L. Bai, S. Zhao, Y. Fu, and Y. Cheng, Chem. Eng. J. 298, 281 (2016). 
[87] N. Di Miceli Raimondi, L. Prat, C. Gourdon, and J. Tasselli, Chem. Eng. Sci. 105, 169 (2014).

[88] Y. Li, R. K. Reddy, C. S. S. R. Kumar, and K. Nandakumar, Biomicrofluidics 8, 54125 (2014).

[89] C. Roizard, Absorption avec réaction chimique (Ed.Techniques Ingénieur, 1997).
[90] Y. Fu, L. Bai, S. Zhao, K. Bi, Y. Jin, and Y. Cheng, Chem. Eng. Sci. 155, 428 (2016).

[91] S. Zhao, A. Riaud, G. Luo, Y. Jin, and Y. Cheng, Chem. Eng. Sci. 131, 118 (2015).

[92] S. G. Ayodele, F. Varnik, and D. Raabe, Phys. Rev. E 80, 016304 (2009). 